\title{
Effects of prenatal stress on lever-press acquisition, delay discounting, and ethanol self -administration in rats
}

Natalie R. Bruner

West Virginia University

Follow this and additional works at: https://researchrepository.wvu.edu/etd

\section{Recommended Citation}

Bruner, Natalie R., "Effects of prenatal stress on lever-press acquisition, delay discounting, and ethanol self -administration in rats" (2010). Graduate Theses, Dissertations, and Problem Reports. 3082.

https://researchrepository.wvu.edu/etd/3082

This Dissertation is protected by copyright and/or related rights. It has been brought to you by the The Research Repository @ WVU with permission from the rights-holder(s). You are free to use this Dissertation in any way that is permitted by the copyright and related rights legislation that applies to your use. For other uses you must obtain permission from the rights-holder(s) directly, unless additional rights are indicated by a Creative Commons license in the record and/ or on the work itself. This Dissertation has been accepted for inclusion in WVU Graduate Theses, Dissertations, and Problem Reports collection by an authorized administrator of The Research Repository @ WVU.

For more information, please contact researchrepository@mail.wvu.edu. 


\title{
Effects of Prenatal Stress on Lever-Press Acquisition, Delay Discounting, and Ethanol Self-Administration in Rats
}

\author{
Natalie R. Bruner \\ Dissertation Submitted to the \\ Eberly College of Arts and Sciences \\ at West Virginia University \\ in partial fulfillment of the requirements \\ for the degree of \\ Doctor of Philosophy \\ in \\ Psychology \\ Karen G. Anderson, Ph.D., Chair \\ Michael Perone, Ph.D. \\ Claire St. Peter Pipkin, Ph.D. \\ Kevin Larkin, Ph.D. \\ Adrienne Salm, Ph.D. \\ Department of Psychology \\ Morgantown, West Virginia \\ 2010
}

Keywords: acquisition; delay discounting; ethanol self-administration; HPA axis; impulsive choice; lever press; prenatal stress; rat 


\begin{abstract}
Effects of Prenatal Stress on Lever-Press Acquisition, Delay Discounting, and

Ethanol Self-Administration in Rats
\end{abstract}

Natalie R. Bruner

It is well established in animal research that the hypothalamic-pituitary-adrenal (HPA) axis plays a central mediating role in the detrimental effects of prenatal stress in mother and offspring. The prenatally stressed (PS) rat exhibits a number of behaviors indicative of increased HPA activity relative to control (CON) rats. Because of these behaviors, PS rats may learn novel responses more quickly than $\mathrm{CON}$ rats. However, there are mixed findings in the literature as to whether prenatal stress facilitates or impedes learning. Experiment 1 was designed to examine effects of prenatal stress on lever-press acquisition using 10-s delays to food pellet delivery in rats. Because of the paucity of literature on the link between stress and impulsive choice, Experiment 2 was designed to assess effects of prenatal stress on delay discounting (impulsive choice) in rats. Previous research has demonstrated that increased stress is correlated with greater facilitation and maintenance of drug taking. There is also evidence that increased rates of delay discounting are correlated with increased drug taking. Experiment 3 was designed to assess effects of prenatal stress on ethanol self-administration in rats. The present set of studies is the first to investigate the variables of stress, delay discounting, and ethanol self-administration using a within-subject design. During Experiment 1, PS rats obtained significantly more food pellets and responded at a higher rates on the lever correlated with food than CON rats. Prenatal stress did not differentially affect impulsive choice (Experiment 2) or ethanol consumption (Experiment 3). As is consistent with past research, a negative correlation was found between indifference points and $\mathrm{g} / \mathrm{kg}$ of ethanol consumed for the lowest ethanol concentration during the two-bottle choice test. Lower indifference points (i.e., greater impulsivity) were correlated with greater $\mathrm{g} / \mathrm{kg}$ of ethanol consumed for one of the testing concentrations. 


\section{Acknowledgements}

I would like to extend a big thank you to the members of my dissertation committee, Karen Anderson, Mike Perone, Claire St. Peter Pipkin, Kevin Larkin, and Adrienne Salm for all of their valuable time and energy that they have contributed to this project. I would especially like to thank my advisor and mentor, Dr. Anderson, for all of her support, honesty, and patience over the past several years. You are truly a great mentor, and you have had a tremendous impact on my development as a scientist and professional (keep up the good work)! I would also like to thank Dr. Adrienne Salm for her collaboration and allowing for us to use her prenatally stressed rat model in the current set of experiments, and her lab technical assistant, Brent Lally, with his assistance with the breeding and prenatally stressing of the rat dams. My deep gratitude goes out to my lab mates, collaborators, and good friends Dr. Jon Slezak, Dr. James Diller, Sally Huskinson, and Christopher Krebs for their support and thoughtful discussions regarding this project. You all are amazing people and researchers, and I am proud to have had the pleasure to work with you as part of the KGA lab team! I would also like to thank my undergraduate advisor, Dr. Jim Dougan, for turning me on the science of behavior with his great enthusiasm for the subject (and life in general). I would like to thank my family (both blood-related and those who have so amiably adopted me as one of their own) for their unconditional love, support, and confidence in me throughout the duration of graduate school. And finally, last but not least, to my incredibly intelligent, rational, and supportive Jon: We've finally made it after five years! I am truly grateful that you were there with me on this journey throughout our graduate school careers. It's been so rewarding being able to share both the ups and downs with you, it's been a rollercoaster ride but we succeeded and reached the finish! 
Table of Contents

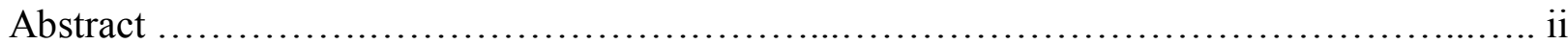

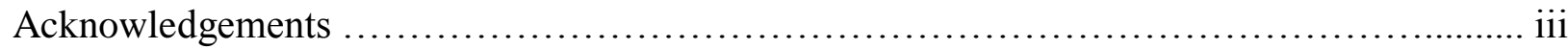

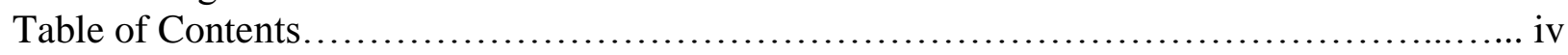

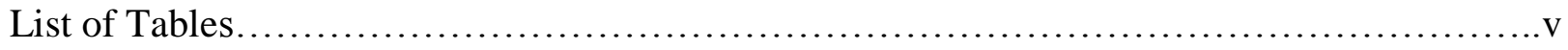

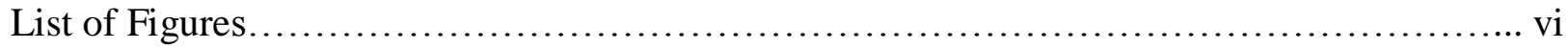

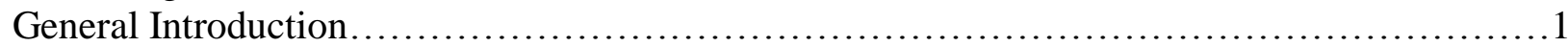

Neurobiology of Prenatal Stress ............................................... 3

Prenatal Stress in Animal Studies.................................................5

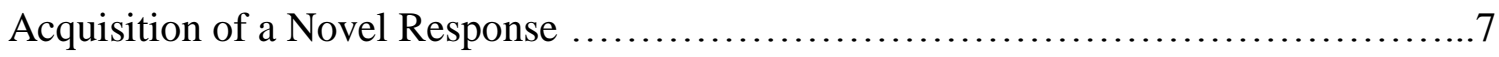

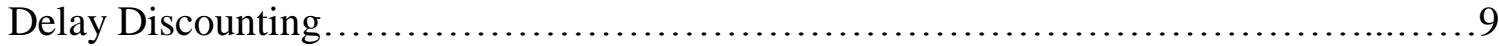

Stress and Drug Self-Administration............................................. 10

Delay Discounting and Drug Self-Administration............................... 12

Statement of the Problem........................................................... 13

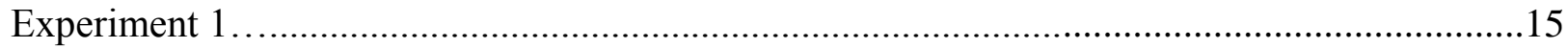

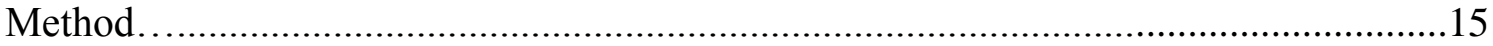

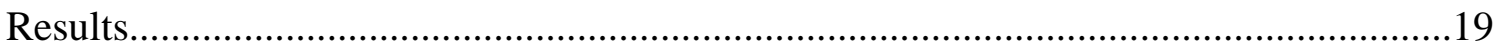

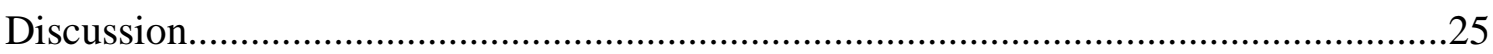

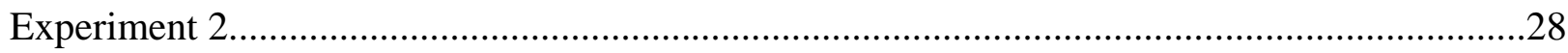

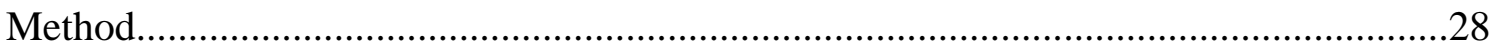

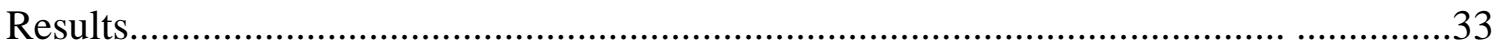

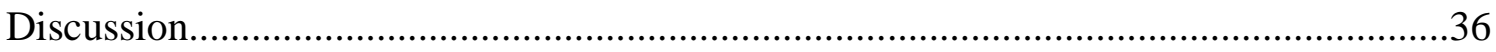

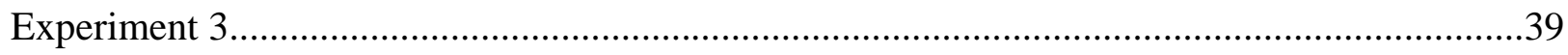

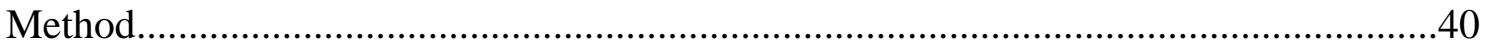

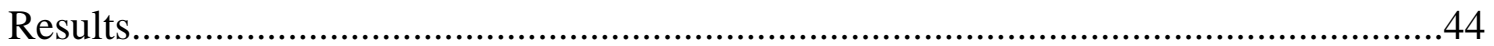

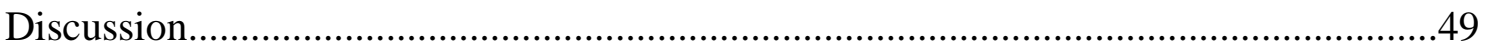

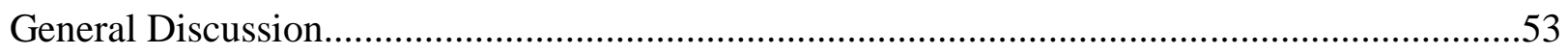

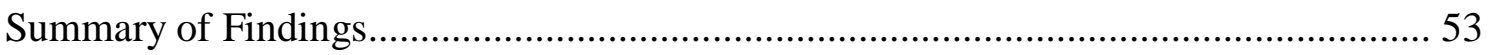

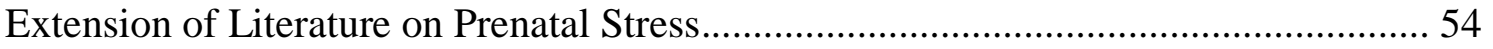

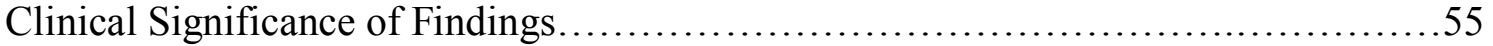

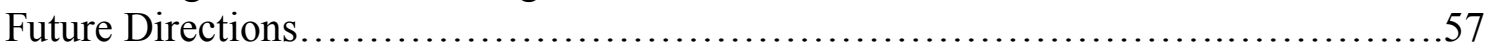

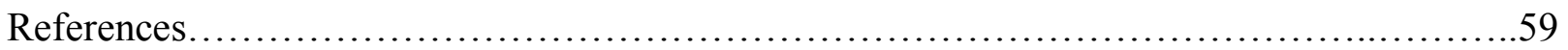




\section{List of Tables}

Table 1: Indifference point (s) for individual subjects during Experiment 2.................35

Table 2: Number of sessions at each sucrose fading concentration during Experiment 3........42

Table 3: Pair-wise comparisons during the sucrose fading procedure in Experiment 3.........46 


\section{List of Figures}

Figure 1: Food pellets and response rates obtained during acquisition.......................21

Figure 2: Cumulative responses on each lever for $\mathrm{CON}$ rats during acquisition................23

Figure 3: Cumulative responses on each lever for PS rats during acquisition...................24

Figure 4: Mean percent choice and indifference points during Experiment 2.................34

Figure 5: Mean $\mathrm{g} / \mathrm{kg}$ ethanol consumed during sucrose fading $\ldots \ldots \ldots \ldots \ldots \ldots \ldots \ldots \ldots \ldots \ldots \ldots$

Figure 6: Mean $\mathrm{g} / \mathrm{kg}$, mls, and percent ethanol consumed during choice test...................48 
General Introduction

Prenatal maternal stress due to negative life events, natural disasters, occupational or other daily stressors, and domestic violence has been linked to premature births, low birth weights, and other negative outcomes such as attention-deficit/hyperactivity disorder (ADHD), cognitive deficits, and developmental disorders in offspring (see Talge, Neal, \& Glover, 2007 for a review). Infants of mothers who reported being exposed to occupational or other daily stressors and anxiety during pregnancy have been found to have lower scores on neurobehavioral assessments and spend less time in active and alert states than infants of mothers who did not experience these stressors (Talge et al.). One study found that mothers who reported higher levels of depression and anxiety during pregnancy had infants who displayed greater negative affect and motor activity when presented with novel toys (Davis et al., 2004). LaPlante et al. (2004) found that toddlers of mothers who were pregnant during a devastating ice storm displayed scores lower than the standardized norm on the Bayley Mental Developmental Index (MDI), which in turn is predictive of poorer reading and spelling abilities and general intellectual functioning later in childhood. Another study found an association between mothers' self-reports of experiencing psychological stress during pregnancy and lower school grades of their offspring at six years of age (Niederhofer \& Reiter, 2004). Prenatal stress has also been associated with an increased likelihood of brain laterality (i.e., mixed-handedness), which has been linked to autism, developmental disabilities, attention problems, schizophrenia and other psychiatric conditions later in life (Glover, O’Connor, Heron, \& Golding, 2004). Van den Bergh et al. (2005) found that adolescents of mothers who experienced higher anxiety during pregnancy exhibited impulsive response patterns during an encoding task (as indicated by quicker response times and more errors made), and had lower scores on an intelligence test compared to adolescents of mothers 
who experienced low-to-moderate anxiety during pregnancy. The most consistently observed outcome of prenatal stress in offspring is ADHD (O'Connor, Heron, Golding, Beveridge, \& Glover, 2002; Van den Bergh et al.; Rodriguez \& Bohlin, 2005). Taken together, these studies suggest that exposure to prenatal stress may result in long-lasting negative outcomes for offspring.

Some related behavioral changes can be modeled in animal research, as is the case in acquisition of a novel response (i.e., learning), delay discounting (i.e., impulsive choice), and self-administration of drugs commonly abused in humans. The present set of studies was designed to assess effects of prenatal stress on these behaviors. In the animal laboratory, the acquisition of a novel response may be examined to investigate the impact of stress on learning. Prenatally stressed (PS) rats have exhibited enhanced conditioned fear and increased behavioral inhibition in response to footshock compared to control (CON) rats (Dickerson, Lally, Gunnel, Birkle, \& Salm, 2005; Griffin, Skinner, Salm, \& Birkle, 2003; Ward, Johnson, Salm, \& Birkle, 2000). It is possible that PS rats may learn novel responses more quickly than CON rats.

Whether this altered learning will extend to a positive reinforcement task may be examined using an acquisition task in which food pellets are delivered following operative lever presses.

Impulsive choice is a major characteristic of several clinical disorders, including ADHD and drug abuse. Impulsive choice may be operationally defined as the choice of a smaller, immediate reinforcer to the exclusion of a larger, more delayed reinforcer, and may have maladaptive consequences. Organisms tend to discount the value of a reinforcer the further in time it is delayed. The procedure first used by Evenden and Ryan (1996) is currently widely used and accepted in the experimental literature for assessing impulsive choice. In this procedure, the delay to the larger reinforcer is increased within session. An indifference point 
may be interpolated by determining the delay value at which choice between the smaller and larger reinforcer is equal. Shorter indifference points are indicative of greater impulsive choice. By studying impulsive choice with such a model, we may examine its relation to drug abuse. High rates of delay discounting have been linked with both the acquisition and maintenance of drug taking in both human and non-human animals (see Setlow, Mendez, Mitchell, \& Simon, 2009 for a review).

Stress has been found to be a major determinant in the acquisition, maintenance, and relapse of drug taking (Sinha, 2001). However, there has been little work investigating a possible link between stress and increased rates of delay discounting. There may be a link between increased delay discounting, stress, and drug self-administration. The present set of studies was designed to investigate effects of prenatal stress on learning, delay discounting, and ethanol selfadministration in rats using a within-subject design.

\section{Neurobiology of Prenatal Stress}

The hypothalamic-pituitary-adrenal (HPA) axis plays a central mediating role in the harmful effects of prenatal stress in both mother and offspring. The HPA axis is active when mammals are adapting to changes in the environment. Typically, the system responds rapidly to stressful stimuli and returns quickly to baseline states of homeostasis. When stimulated, neurons in the paraventricular nucleus of the hypothalamus (PVN) secrete corticotropin releasing factor (CRF), which induces production of adrenocorticotropin hormone (ACTH) from the pituitary gland. ACTH is released into the systemic circulation and stimulates release of glucocorticoids (e.g., cortisol in humans and corticosterone in rats), epinephrine, and norepinephrine. Elevated levels of glucocorticoids in the serum prepare the body for an adaptive stress response, and also begin to interact with corticoid receptors to inhibit or terminate the stress response through 
negative feedback. Two steroid receptors mediate negative feedback to the brain (Welberg \& Seckl, 2001). Dysfunction in either receptor system may result in higher and more enduring levels of glucocorticoids, which could impede the organism's ability to adapt to its environment.

Changes in dopamine (DA) neurotransmission have also been implicated in the behavioral differences in offspring exposed to prenatal stress relative to offspring not exposed to prenatal stress. DA levels in the forebrain play a role in such functions as motor integration, choice, learning, and memory (Finlay \& Zigmond, 1997). Control rats have asymmetrical levels of neurotransmitters that are related to brain organization, which in turn is crucial to several behavioral functions (Adrover, Berger, Perez, Tarazi, \& Antonelli, 2007). Asymmetrical levels of several neurotransmitters, including DA, have been found in the nucleus accumbens, cortex, and striatum of normal rats (Rosen, Finklestein, Stoll, Yutzey, \& Denenberg, 1984). More specifically, the authors found a greater concentration of DA in the right cortex and accumbens of the rat. Adrover et al. found that prenatal stress increased D2 receptors in both the left and the right hemispheres of the nucleus accumbens, and the left-right asymmetry of the D2 receptors was lost in PS rats. The authors suggest that because DA plays a role in impulsivity, this loss of asymmetry may contribute to behavioral and cognitive impairments including ADHD and depression. Thus, PS rats may emit greater impulsive choice relative to CON rats on a delay discounting task.

The PS rat has been found to be a valid model for significant aspects of anxiety disorders (Dickerson et al., 2005). By exposing rat dams to stressors during pregnancy (i.e., exposure to a novel environment and subcutaneous injections of saline), developing fetuses were exposed to high plasma levels of endogenous glucocorticoids, resulting in altered neurobiology and behaviors that persisted late into adulthood of offspring. Adult PS rats have a hyperactive HPA 
axis, as is reflected in higher levels of serum corticosterone following application of stressors. The serotogenic and noradregenic neurotransmitter systems are also altered in PS rats, as is evident by the greater levels of norepinephrine and serotonin following stressors than control rats. Salm et al. (2004) found that these changes are also accompanied by altered amygdala development. Thus, the PS rat is a valid animal model and may be a useful tool for exploring the role of stress on other behaviors, such as learning, impulsive choice, and drug selfadministration.

\section{Prenatal Stress in Animal Studies}

Due to inherent problems in human research, investigators have focused on non-human animal work to investigate the mechanisms underlying the long-term effects of prenatal stress. Much of the research conducted has found evidence of increased HPA-axis activity, as well as behavioral differences in the offspring exposed to prenatal stress (see Weinstock, 1996 for a review). For example, Weinstock, Matlina, Maor, Rosen, and McEwen (1992) exposed rat dams to noise and light stress throughout pregnancy. When placed in an open field (an environment which is aversive to rats), offspring of the dams that were exposed to stress exhibited higher and more prolonged serum corticosterone responses compared to CON rats. PS rats also made fewer entries into the center of the open field and voided a greater number of fecal pellets during the session. A study conducted by Vallee et al. (1997) with offspring of dams that had been exposed to restraint stress during the last week of pregnancy yielded similar results. They found that PS rats exhibited prolonged stress-induced plasma corticosterone secretion, as well as heightened behavioral responses to novel stimuli, including freezing, less time spent exploring, and increased defecation. PS rats have exhibited a number of "fearful" behaviors in several other studies, including increased inhibition in response to acute footshock, decreased time spent in the 
center of an open field, less time spent in open arms of the elevated-plus maze, increased defensive withdrawal, and enhanced conditioned fear relative to CON rats (Dickerson et al., 2005; Griffin et al., 2003; Poltyrev, Keshet, Kay, \& Weinstock, 1996; Ward et al., 2000). In addition, it has been found that these fearful behaviors may be ameliorated following administration of CRF antagonists (Ward et al.). More specifically, administration of CRF antagonists abolished the behavioral differences observed between PS and CON rats following restraint stress in a defensive withdrawal test.

There has been little research conducted investigating whether the offspring of PS rats exhibit heightened HPA-axis responses in baseline conditions (i.e., in the absence of an acute stressor). Using chronic indwelling venous catheters in rats, Koehl et al. (1999) found that there were no differences in daily basal corticosterone levels throughout most of the day. However, the daily peak response of corticosterone was higher and occurred sooner in PS rats relative to CON rats. The present set of studies examined effects of prenatal stress on learning, impulsive choice, and ethanol self-administration in the absence of an acute stressor.

Although the majority of the research on prenatal stress had been done with rodents, there has been limited work conducted with nonhuman primates. Clarke, Wittwer, Abbot, and Schneider (1994) collected blood samples from PS and CON juvenile rhesus monkeys following stressors and in the absence of stressors (i.e., during baseline). The authors found that PS monkeys had significantly higher cortisol levels compared to CON monkeys at baseline, as well as higher adrenocorticotropic hormone (ACTH) levels following exposure to stressors. Research conducted by Schneider and colleagues have found that the juvenile offspring of rhesus monkeys exposed to a daily acoustical startle protocol (i.e., three 1-s broadcasts of a horn at 1-4 min intervals) while pregnant exhibited long-term adverse behavioral responses to reunion with peers 
following isolation stress (Schneider, Moore, Kraemer, Roberts, \& DeJesus, 2002; Schneider, 1992; Clarke \& Schneider, 1993). More specifically, the PS monkeys exhibited significantly less focused exploration and greater stereotypical movements (e.g., pacing) relative to CON monkeys (i.e., offspring of mothers undisturbed during pregnancy). The PS monkeys also tended to cling more to their peers than CON monkeys. Coe et al. (2003) found that these altered behaviors were accompanied by significantly higher plasma cortisol levels in the PS monkeys relative to the CON monkeys. Schneider and Coe (1993) stressed pregnant squirrel monkeys by repeatedly placing them in a novel social group and home environment throughout pregnancy. This disruption in the mother's social relationships adversely affected the offspring. The infants exposed to prenatal stress exhibited impairments in motor abilities (i.e., poorer balancing ability and motor maturity) compared to infants of mothers not exposed to prenatal stress. The PS monkeys also exhibited a shorter attention span and shorter duration of orientation (i.e., looking) when exposed to a novel plastic toy relative to CON monkeys. Thus, prenatal stress affects behavior on many levels, including development of motor skills, social relationships, attention deficits, and learning. The present set of studies was designed to investigate effects of prenatal stress on some relevant behaviors (i.e., learning, impulsive choice, and ethanol selfadministration).

\section{Acquisition of a Novel Response}

Because of the enhanced conditioned fear and increased behavioral inhibition exhibited by PS rats, PS rats may have greater sensitivity to behavioral consequences than CON rats. PS rats may thus learn novel responses more quickly than $\mathrm{CON}$ rats, when using aversive conditioning. However, research using operant tasks has demonstrated that rodents exposed to prenatal stress display learning impairments during spatial learning tasks (Vallee et al., 1999; 
Kapoor, Kostaki, Janus, \& Matthews, 2009). For example, Vallee et al. found that PS rats spent less time in a novel arm of a Y-maze and made more errors during a radial maze task compared to $\mathrm{CON}$ rats. Kapoor et al. found that PS guinea pigs exhibited deficits in spatial learning using a water maze (i.e., PS guinea pigs covered more distance in the water maze before finding a hidden platform than CON guinea pigs). Although research findings have indicated that exposure to prenatal stress enhances the association between aversive stimuli and elicited behavior (e.g., freezing, defecating) in aversive conditioning tasks, operant research conducted with PS and $\mathrm{CON}$ animals has found that exposure to prenatal stress impedes learning. Experiment 1 of the present set of studies was designed to further assess effects of prenatal stress on operant behavior using a lever-press acquisition procedure.

It is well established that the rate or frequency of an established response may be decreased when the reinforcer maintaining it is delayed (cf. Lattal, 2010; Mazur, 1987). Previous research has demonstrated that a new behavior may be established when reinforcement is not immediate or signaled following a response, and the response is not explicitly shaped (e.g., Lattal \& Gleeson, 1990; Anderson \& Elcoro, 2007). Acquisition of novel responses using delayed reinforcement has been demonstrated with various species and response topographies. Lattal and Gleeson found that key-pecking in pigeons and lever-pressing in rats may be established using 10 to 30-s delays to food reinforcement. Anderson and Elcoro demonstrated that Lewis and some Fischer 344 rats acquired lever pressing using 20-s delays to food reinforcement. By using such a procedure, how effects of delayed consequences on the acquisition of a novel behavior differ as a result of individual differences (e.g., behavioral history, sex, genetics, and prenatal stress) may be examined. Experiment 1 of the present set of studies was designed to evaluate lever-press acquisition with a 10-s delay to food reinforcement in PS and CON rats. 


\section{Delay Discounting}

According to the American Psychiatric Association Diagnostic Manual-IV-TR (APA DSM-IV, 2000), several clinical disorders are characterized by impulsivity. Among these disorders are drug abuse, ADHD, aggression, and pathological gambling. Extending knowledge in the area of impulsive choice may lead to the development and improvement of methods for treating these disorders. In the experimental laboratory, impulsive choice is often studied using a delay-discounting procedure. In such a procedure, the subject is presented with a choice for a smaller, immediate reinforcer (impulsive choice) and a larger, more delayed reinforcer (selfcontrolled choice). Choice for the smaller reinforcer to the exclusion of the larger reinforcer results in loss of overall reinforcement, and thus may be considered to be maladaptive. This procedure may model some maladaptive behaviors in human substance abusers, such as choosing an immediate "high" over a more delayed outcome of greater health benefits that accompany drug abstinence.

The value of outcomes tend to be discounted as a function of a delay to their presentation, such that consequences become more ineffective in controlling behavior the further in time that they are displaced. For instance, given a choice between $\$ 10$ now and $\$ 100$ now, most people would choose $\$ 100$ now. However, if given a choice between $\$ 10$ now and $\$ 100$ in one year, many people would still choose $\$ 10$ now, despite the increase in reinforcer magnitude. The addition of a delay to presentation weakens the reinforcing effect (value) of the larger reinforcer. The hyperbolic delay-discounting function offers a quantitative account of this phenomenon (Mazur, 1987). The procedure first used by Evenden and Ryan (1996), in which the delay to the larger reinforcer is increased within session, is one that is widely used. An indifference point between two outcomes may be interpolated by determining the delay value at which choice 
between the smaller and larger reinforcer is equal. Longer indifference points indicate more selfcontrolled choice, and shorter indifference points indicate more impulsive choice. This delaydiscounting procedure is frequently used in experimental literature, and is considered to be a useful tool in studying impulsive behavior.

Little work has been done investigating effects of stress on delay-discounting tasks. White, Lawford, Morris, and Young (2009) found that exposing humans who possessed a genotype associated with decreased striatal binding of D2 receptors, as well as working memory impairments, to acute stressors (i.e., participants were asked to prepare a 5-min speech on their least favorite body part) resulted in elevated measures of impulsive choice, relative to those participants not exposed to the stressor and participants not possessing the phenotype. Fields, Leraas, Collins, and Reynolds (2009) found that higher rates of delay discounting may mediate the relation between stress and tobacco use in adolescents. More specifically, adolescent smokers self-reported higher levels of stress, and had higher rates of delay discounting for hypothetical money rewards relative to non-smoking control participants. More research is needed to examine a possible link between higher rates of delay discounting and stress. Experiment 2 of the present set of studies was designed to assess effects of prenatal stress on delay discounting in rats.

\section{Stress and Drug Self-Administration}

Experimental research with humans has found that acute and chronic stress plays an important role in increasing vulnerability to drug use and relapse in substance abusers (Sinha, 2001). One model postulates that stress may lead to changes in the brain reward system, resulting in greater sensitivity to the reinforcing properties of drugs (Koob \& Le Moal, 1997). The mechanisms underlying stress and drug abuse remain unclear, however, and future research is needed in order to better understand the factors mediating this association. 
Studies have been conducted linking indicators of stress to both acquisition and maintenance of drug self-administration. Mantsch, Saphier, and Goeders (1998) found that administration of the stress hormone corticosterone facilitated acquisition of cocaine selfadministration in rats. Rats that were treated prior to the experimental session with corticosterone $(2.0 \mathrm{mg} / \mathrm{kg}$, i.p.) acquired intravenous cocaine self-administration at a lower dose than vehicletreated control rats. West and Weiss (2006) found that rats selectively bred for vulnerability to stress self-administered significantly more ethanol than rats that were bred for resistance to stress and non-selectively bred rats. Vulnerability to stress was measured by a relatively short duration of "struggling" and increased immobility when exposed to a forced-swim test, and these rats were designated swim-test susceptible (SUS). SUS rats consumed amounts of ethanol similar to that of rats bred specifically for alcohol intake (alcohol-preferring rats). Taylor, Harris, and Vogel (1990) found that rats selectively bred for high plasma catecholamines (e.g., epinephrine and norepinephrine) stress responses to immobilization consumed more ethanol solution (5-10\% $\mathrm{v} / \mathrm{v})$ and cocaine solution $(0.02 \% \mathrm{v} / \mathrm{v})$ than rats that were selectively bred as low catecholamine responders. Selectively breeding for susceptibility to stress and administration of the stress hormone corticosterone have been correlated with facilitated acquisition and higher levels of drug self-administration in rats. Other methods of inducing stress in laboratory animals have successfully resulted in higher levels of drug self-administration. Among these are repeated tail pinch (Piazza, Deminiere, Le Moal, \& Simon, 1990), exposure to inescapable footshock (Goeders \& Guerin, 1994), and isolation housing (Schenk, Lacelle, Gorman, \& Amit, 1987). Previous research has also shown that exposure to prenatal stress is linked to increased sensitivity to effects of experimenter-administered amphetamine (Deminiere et al., 1992; Henry et al., 1995) and cocaine (Kippin, Szumlinski, Kapasova, Rezner, \& See, 2008). Facilitated 
acquisition of cocaine self-administration (Thomas, Hu, Lee, Bhatnagar, \& Becker, 2009) and reinstatement of cocaine-seeking behavior following extinction (Kippin et al.) in PS rats has also been observed.

Little work has been done investigating effects of prenatal stress on ethanol selfadministration in rats. DeTurck and Pohorecky (1987) observed that rats exposed to prenatal stress showed significantly attenuated responses to acute alcohol (i.e., decreases in body temperature, motor coordination, and startle amplitude) compared to control rats. Darnaudery et al. (2007) found that exposing alcohol-preferring rats to intense stressors (i.e., shock) reduced ethanol self-administration in rats that had not been exposed to prenatal stress. Ethanol selfadministration did not decrease in rats that had been exposed to prenatal stress. More research is needed to investigate effects of prenatal stress on ethanol self-administration. Experiment 3 of the present set of studies was designed to assess effects of prenatal stress on ethanol-self administration in rats.

\section{Delay Discounting and Drug Self-Administration}

Human drug abusers often discount the value of a delayed reinforcer at a higher rate than non-drug-using control participants. For instance, Mitchell, Fields, D’Esposito, \& Boettiger (2005) found that alcoholics discounted the value of a delayed hypothetical reward more than nonalcoholic control participants. Another study with nicotine abusers found that current smokers discounted health gains and losses at a steeper rate than never smokers (Odum, Madden, \& Bickel, 2002). Field, Christiansen, Cole, and Goudie (2007) found that adolescent heavy drinkers discounted the value of delayed hypothetical alcohol at a steeper rate than lighterdrinking adolescents. 
There is a well-established relation between greater impulsivity and drug selfadministration in the non-human animal literature (see Setlow et al., 2009 for a review). For example, Logue, Swartz, and Wehner (1998) demonstrated a correlation between performance on a response inhibition task and ethanol self-administration in laboratory mice. The operational definition of impulsivity used was the inability to withhold a nosepoke response (i.e., inserting of the nose through a hole in the operant-chamber wall). Mice that were found to be more impulsive consumed more ethanol (3-10\% v/v) than the more self-controlled mice. Perry, Larson, German, Madden, \& Carroll (2005) found that rats with greater rates of delay discounting acquired cocaine self-administration more quickly than rats with less steep rates of delay discounting.

The above studies, and those conducted with human substance abusers, suggest a correlation between increased rates of delay discounting and drug self-administration or reinforcement. As part of the present analyses, within-subject correlations between delay discounting (Experiment 2) and ethanol self-administration (Experiment 3) were conducted. The present set of studies was designed to allow for within-subject comparisons between prenatal stress, and delay discounting and ethanol self-administration.

\section{Statement of the Problem}

Prenatal maternal stress due to daily stressors, negative life events, natural disasters, occupational stressors, and domestic violence has been linked to premature births, low birth weights, and other negative outcomes in offspring. Some related behavioral changes can be modeled in animal research, as is the case in acquisition of a novel response, impulsive choice, and self-administration of drugs commonly abused in humans. It is well established in nonhuman animal research that the HPA axis plays a central mediating role in the detrimental effects of prenatal stress in mother and offspring. The PS rat has been found to exhibit a number of 
behaviors indicative of increased HPA activity, including enhanced conditioned fear, increased behavioral inhibition in response to footshock, decreased time spent in the center of an open field, and increased defensive withdrawal relative to CON rats (Dickerson et al., 2005; Griffin et al., 2003; Ward et al., 2000). Because of these behaviors, PS rats may learn novel responses more quickly than $\mathrm{CON}$ rats. However, there are mixed findings in the literature as to whether prenatal stress facilitates or impedes learning. To date, no studies have examined effects of prenatal stress on lever-press acquisition. Experiment 1 was designed to examine effects of prenatal stress on lever-press acquisition using 10-s delays to food pellet delivery in rats.

Little work has been done investigating effects of stress on measures of impulsivity. The work that has been done has found a link between increased measures of stress and elevated measures of impulsive choice (White et al., 2009; Fields et al., 2009). Experiment 2 of the present set of studies was designed to assess effects of prenatal stress on delay discounting in rats.

Experimental research with both humans and non-human animals has found that acute and chronic stress plays an important role in increasing vulnerability to drug use and relapse of drug taking (Sinha, 2001; Mantsch et al., 1998; West \& Weiss, 2006). Little work has been done investigating effects of prenatal stress on ethanol self-administration in rats. One study found that exposing alcohol-preferring rats to intense stressors (i.e., shock) reduced ethanol selfadministration in CON rats, but not in PS rats (Darnaudery et al., 2007). Experiment 3 was designed to assess effects of prenatal stress on ethanol self-administration in rats.

The correlation between steeper rates of delay discounting and substance abuse has been well established (see Reynolds, 2006 for a review). However, the link between stress, impulsive choice, and drug-self-administration has not yet been investigated using a within-subject design. 
Examining delay-discounting functions and ethanol self-administration in the PS rat may shed more light on the correlation between substance abuse, impulsive behavior, and stress. Should it be found that higher levels of delay discounting correlate with enhanced drug selfadministration, implications may be made about neurobiological variables underlying mechanisms of stress, impulsive behavior, and drug abuse.

\section{Experiment 1}

Experiment 1 was designed to assess effects of prenatal stress on learning using a positive reinforcement task in rats. There have been mixed results in the literature as to whether prenatal stress facilitates or impedes learning. To further investigate effects of prenatal stress on learning, a lever-press acquisition procedure with a 10-s delay to food reinforcement was used. First, a single feeder training session in which food pellets were delivered according to a variable-time (VT) 60-s schedule was conducted. Subjects were then exposed to a single 8-h lever-press acquisition session in which a tandem fixed-ratio (FR) 1 differential-reinforcement-of-otherbehavior (DRO) 10-s schedule of reinforcement was programmed for responses on one of the two levers. A single response on the lever associated with reinforcement (operative lever) resulted in a 10-s delay to food pellet delivery. Responses on either lever during the delay reset the delay to ensure that $10 \mathrm{~s}$ always elapsed between a response and food pellet delivery.

\section{Method}

\section{Prenatal Stress Procedure}

For those rats in the PS group, pregnant dams were exposed to acute stressors at West Virginia University's Health Sciences Center following an established procedure similar to that of Griffin et al. (2003). Beginning on gestational day 14, pregnant dams were removed from their homecages, and briefly placed in a novel cage. Rats then received a subcutaneous injection of 0.1 
$\mathrm{ml} 0.9 \%$ saline before being returned to their homecages. This procedure was repeated once daily at random times of the day, and by different lab personnel, until the pups were born (about gestational day 22). For rats in the CON group, dams were left undisturbed throughout pregnancy, except for routine animal husbandry. Male pups were weaned from the dam on postnatal day 22, and transferred to the West Virginia University Life Sciences Building. Subjects were allowed free access to food and water until 50 days after birth. Thereafter, they were fed approximately $15 \mathrm{~g}$ of food daily. Behavioral testing began 60-70 days after birth.

The first time the rats were bred, an insufficient number of male offspring were born (i.e., three PS rats and six CON males). These subjects from litter A were PSa-1, PSa-2, PSa-3, CONa-1, CONa-2, CONa-3, CONa-4, CONa-5, and CONa-6. Because it was necessary to breed the dams again, two dams were again exposed to the prenatal stressing procedure about two months after the first litter was born. One dam was again in the control group. The second litter of rats (litter B) yielded an additional five PS rats and two CON rats. These subjects were labeled PSb-1, PSb-2, PSb-3, PSb-4, PSb-5, CONb-1, and CONb-2.

\section{Subjects}

Eight experimentally naïve PS male rats and eight experimentally naïve CON male rats (Robert C. Byrd Health Sciences Center, Morgantown, WV) served as subjects in Experiment 1. Subjects were housed individually with free access to water in their home cages. Temperature and humidity were maintained at constant levels and a reverse 12 -h light-dark cycle was in effect. Sessions were conducted at approximately the same time each day. Subjects were fed approximately $15 \mathrm{~g}$ of food no less than $30 \mathrm{~min}$ following each experimental session, unless otherwise stated. This schedule resulted in approximately 22 hours of food restriction prior to the 
start of each session. The food restriction regimen was in accordance with NIH and Institutional Guidelines and was approved by the West Virginia University Animal Care and Use Committee. Apparatus

Sessions were conducted in eight standard operant-conditioning chambers for rats, each enclosed in a melamine sound-attenuating cubicle (Med Associates, VT). Each chamber contained a working area of $30.5 \times 24.1 \times 21.0 \mathrm{~cm}$, a grid floor, and a $45-\mathrm{mg}$ pellet dispenser with a pellet receptacle centered between two retractable response levers. The levers were $11.5 \mathrm{~cm}$ apart from each other, $4.7 \mathrm{~cm}$ wide, protruded $2.3 \mathrm{~cm}$ into the chamber, elevated $6 \mathrm{~cm}$ from the grid floor and required a force of at least $0.25 \mathrm{~N}$ for a response to be recorded. Two $28-\mathrm{V}$ stimulus lights of $2.5 \mathrm{~cm}$ in diameter were approximately $7 \mathrm{~cm}$ above each lever. During sessions, a white houselight opposite the wall containing the operandum was illuminated, as well as the lights above each lever. A ventilation fan served to circulate air and mask extraneous noise. Equipment was interfaced to a computer and experimental sessions and data collection was programmed and conducted with MedPC-IV (Med Associates, VT).

\section{Magazine Training}

All subjects were first exposed to a single 1-h session of feeder training. Subjects were food restricted for $22 \mathrm{~h}$ before the start of the feeder-training session. At the beginning of the session, each rat was placed in a darkened operant-conditioning chamber. Immediately after, the ventilation fan was turned on and the houselight was illuminated. The response levers were retracted from the chamber for the duration of this session. Food pellets were delivered according to a variable-time (VT) 60-s schedule. Values for the VT were obtained using a Fleshler-Hoffman sequence generator for 20 cycles of reinforcer delivery and repeated three times. Pellet delivery was signaled by a 0.5 -s flash of the houselight. The session ended 
following the delivery of 60 food pellets. The food receptacles were checked for uneaten food pellets. If a significant number of food pellets were not consumed (i.e., more than six food pellets were present), additional feeder training sessions were conducted. Sessions were conducted on adjacent days for each group (PS or CON). Following completion of feeder training, rats were restricted from food for $48 \mathrm{~h}$ prior to the start of the acquisition session.

\section{Acquisition}

A single 8-h lever-press acquisition session was conducted on adjacent days following feeder training for each group. Each rat was placed in a darkened operant-conditioning chamber. Immediately after, the ventilation fan was turned on, the response levers were extended into the chamber, and the houselight and stimulus lights above the levers were illuminated. A tandem FR 1 DRO 10-s schedule of reinforcement was programmed for responses on one of the two levers. A single response on the lever associated with reinforcement (operative lever) resulted in a 10-s delay to food pellet delivery. Responses on either lever during the delay resulted in resetting the delay. This ensured that the delay between the last response on the operative lever and food delivery was always a minimum of $10 \mathrm{~s}$. The operative lever was counterbalanced within groups. Responses on the other lever (inoperative lever) were recorded but had no other scheduled consequences except resetting the delay. Responses on each lever and the total number of food pellets obtained were recorded during the session.

\section{Data Analysis}

Statistical analyses of the data were used to determine significant differences between groups on measures of body weight, response rate on each lever, number of responses during the delay, and food pellets obtained (One-Way Analysis of Variance; ANOVA). Response rates were calculated by dividing the total number of responses on each lever by total session time 
(responses per minute; rpm). The time of lever-press acquisition was quantitatively defined by the time when $25 \%$ of the total number of responses occurred on the operative lever. Sessions were divided into 1-min bins, and the bin in which $25 \%$ of the total responses were reached was identified. Standard errors around the mean (SEM) were calculated for all data presented. In addition, cumulative records were visually assessed for evidence of response acquisition as characterized by positively accelerated curves.

\section{Results}

At the start of Experiment 1, the PS rats weighed an average of $263.8 \mathrm{~g}(S E M=6.74 \mathrm{~g})$, and the CON rats weighed an average of $246.1 \mathrm{~g}(S E M=6.42 \mathrm{~g})$. This difference in weight was not statistically significant $[f(1,15)=3.59, \mathrm{p}=0.079]$. For the CON rats, rats in litter $\mathrm{A}(M=$ $254.17 \mathrm{~g}, S E M=5.00 \mathrm{~g})$ weighed more than rats in litter B $(M=222.00 \mathrm{~g}, S E M=1.00 \mathrm{~g})$. This difference was statistically significant, $[f(1,7)=12.37, \mathrm{p}=0.013]$. For the PS rats, rats in litter $\mathrm{A}(M=282.33 \mathrm{~g}, S E M=6.84 \mathrm{~g})$ weighed more than rats in litter $\mathrm{B}(M=252.60 \mathrm{~g}, S E M=5.52$ g). This difference was statistically significant, $[f(1,7)=11.18, \mathrm{p}=0.016]$. Because all rats in the PS and CON groups were observed to have consumed at least $95 \%$ of the food pellets delivered during the feeder training session, no additional feeder training sessions were required.

Figure 1 (top panel) shows the mean food pellets obtained for the PS and CON groups during the acquisition session. On average, subjects in the PS group obtained almost three times the number of food pellets $(M=277.6$ pellets, $S E M=23.7$ pellets $)$ earned by subjects in the CON group $(M=94.8$ pellets, $S E M=45.6$ pellets $)$. This difference between groups was statistically significant, $[f(1,15)=12.66, \mathrm{p}=0.003]$.

Figure 1 (middle panel) shows mean response rates on the operative lever for both groups. Subjects in the PS group also responded on the operative lever $(M=0.77 \mathrm{rpm}, S E M=$ 
$0.08 \mathrm{rpm})$ at twice the rate as subjects in the CON group $(M=0.33 \mathrm{rpm}, S E M=0.17 \mathrm{rpm})$. This difference between groups was statistically significant, $[f(1,15)=5.64, \mathrm{p}=0.032]$.

Figure 1 (bottom panel) shows mean response rates on the inoperative lever for both groups. There were no statistically significant differences between groups with response rates on the inoperative lever. In addition, there were no statistically significant differences between PS and $\mathrm{CON}$ rats with the absolute number of responses emitted on both levers during the delay to food pellet delivery. 

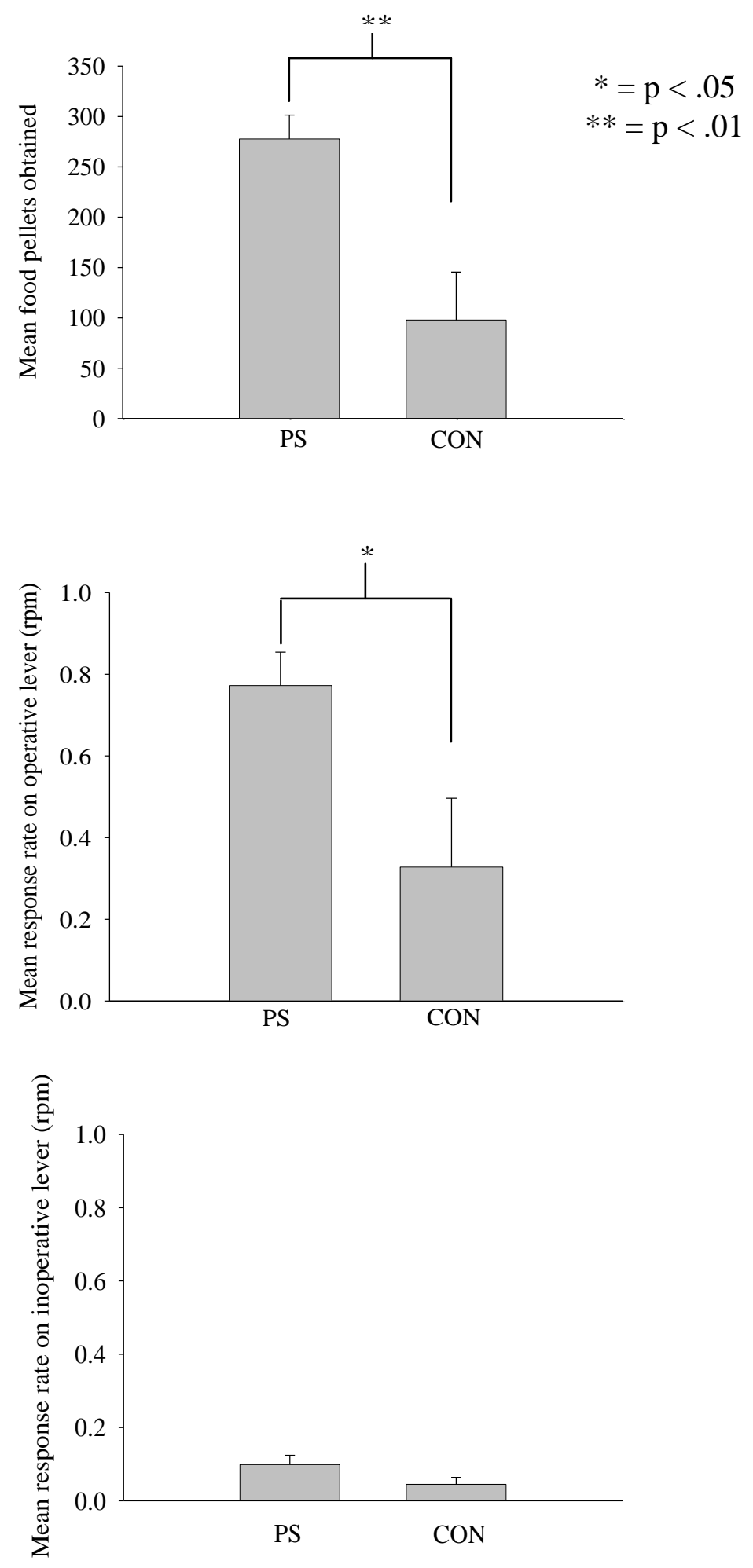

Figure 1. Mean food pellets earned (top panel), mean response rate on the operative (middle panel) and inoperative lever (bottom panel) during acquisition. SEM for all data are presented. 
There were no differences between litters A and B with food pellets obtained or response rates on the operative lever during the acquisition session. However, the $\mathrm{CON}$ rats in litter A emitted lower response rates on the inoperative lever $(M=0.017 \mathrm{rpm}, S E M=0.006 \mathrm{rpm})$ than $\mathrm{CON}$ rats in litter B $(M=0.128 \mathrm{rpm}, S E M=0.015 \mathrm{rpm})$. This difference was statistically significant, $[f(1,7)=65.18, \mathrm{p}=0.00]$. There were no differences found between PS rats in litters $\mathrm{A}$ and $\mathrm{B}$ with response rates on the inoperative lever.

Visual inspection of the cumulative response records (Figures 2 and 3) shows that only three of the $\mathrm{CON}$ rats acquired the response (CONa-3, $\mathrm{CONb}-1$, and $\mathrm{CONb}-2)$, and all eight of the PS rats acquired the lever press response. On average, for those subjects in the PS group that acquired the lever-press response, the 60 -s bin which $25 \%$ of the total responses were emitted was slightly later in the session $(M=181.4$ minutes, $S E M=36.6$ minutes $)$ than those in the CON group $(M=160.7$ minutes, $S E M=44.2$ minutes $)$. This difference between groups was not statistically significant, $[f(1,15)=0.97, \mathrm{p}=0.762]$. 

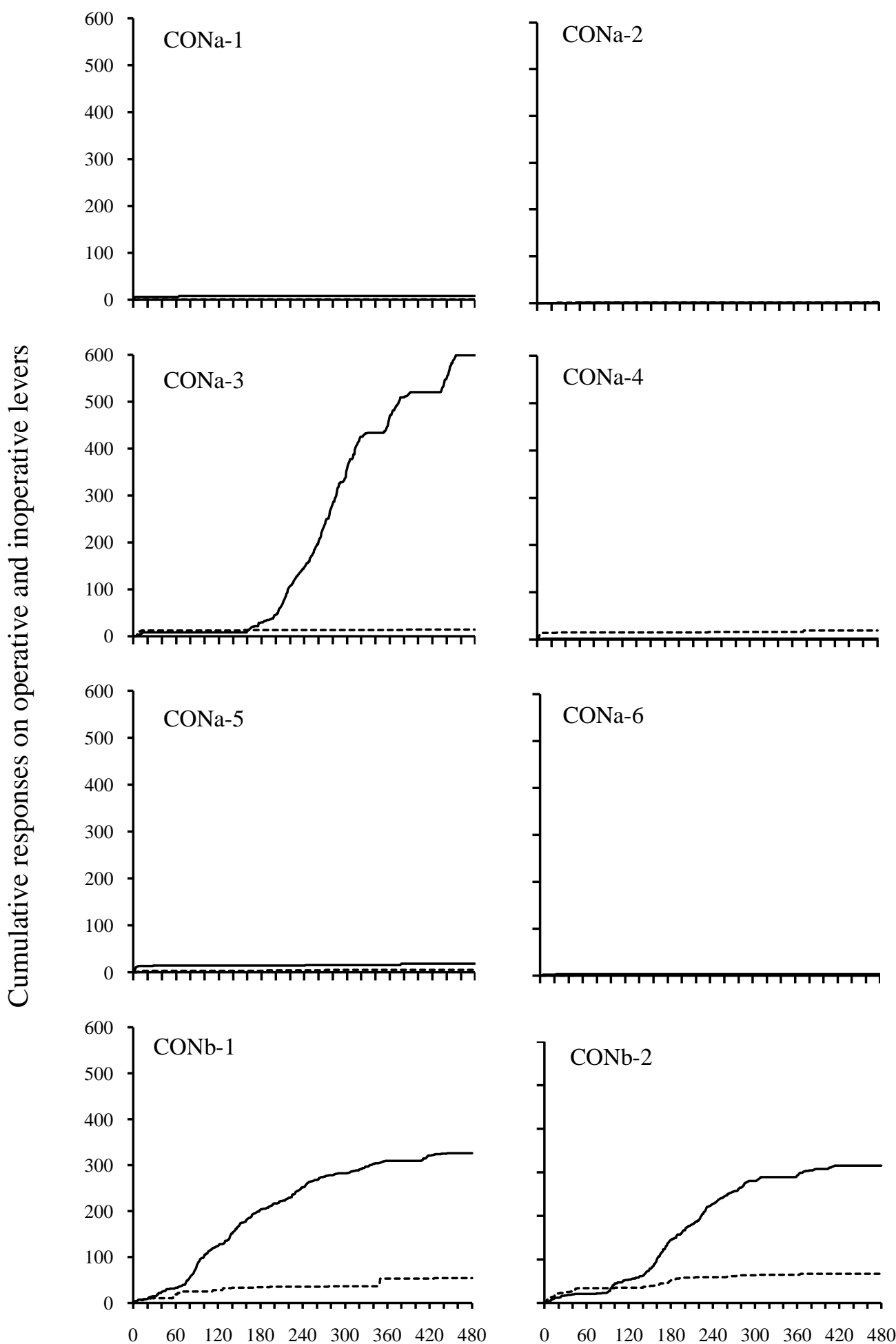

Minutes

Figure 2. Cumulative responses on operative (solid line) and inoperative (dashed line) levers by individual CON rats during the 8-h acquisition session. 

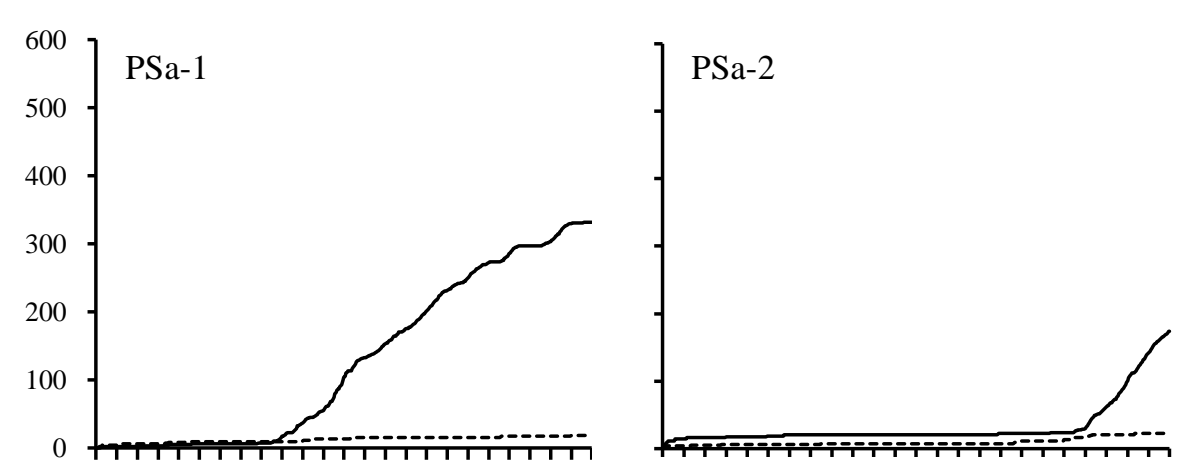

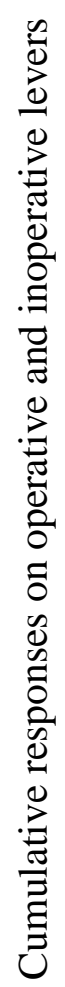
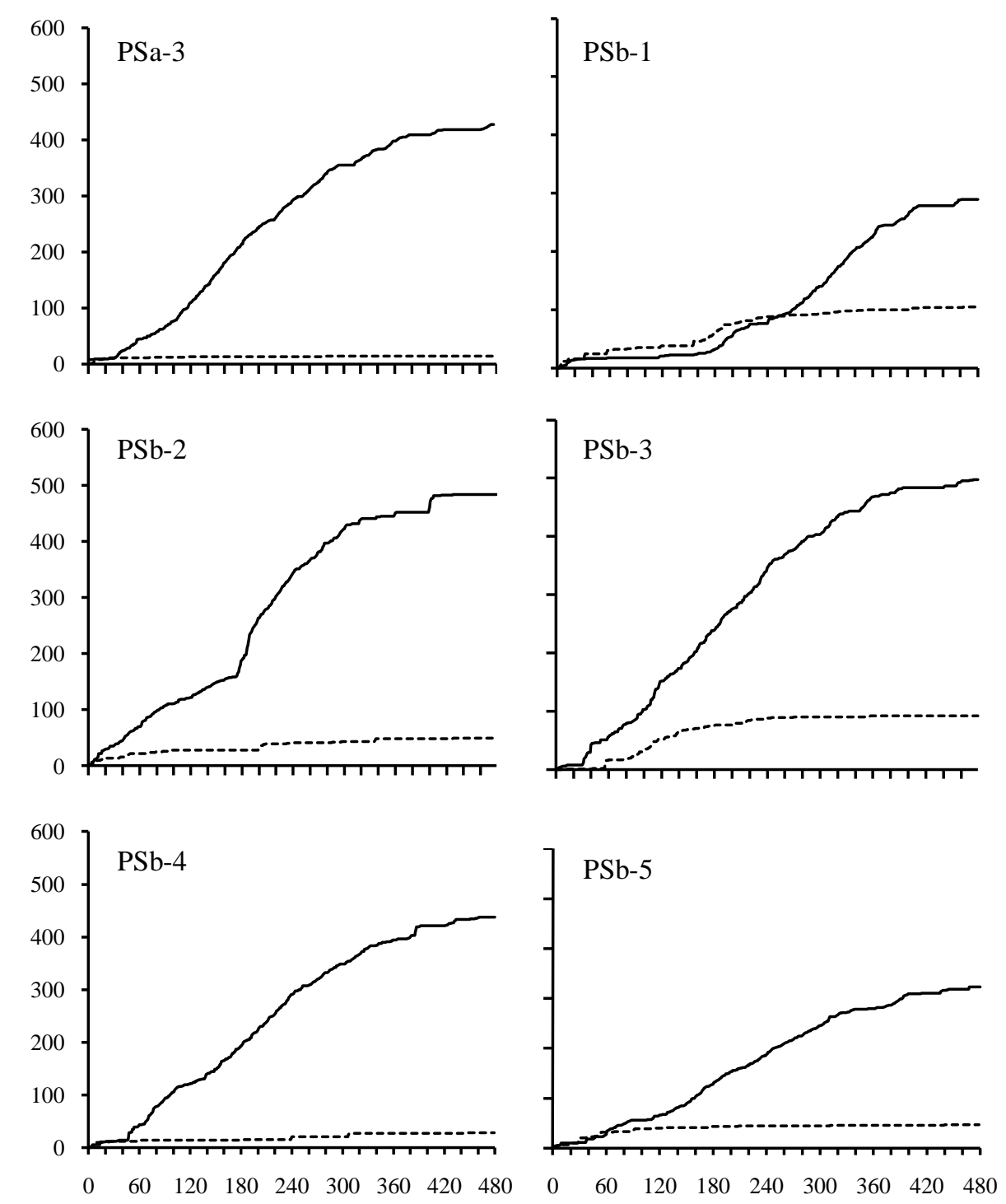

Minutes

Figure 3. Cumulative responses on operative (solid line) and inoperative (dashed line) levers by individual PS rats during the 8-h acquisition session. 


\section{Discussion}

During Experiment 1, effects of prenatal stress on lever-press acquisition using 10-s delays to food reinforcement in rats were investigated. The subjects in the PS group obtained significantly more food pellets and emitted significantly higher response rates on the operative lever relative to subjects in the CON group during the acquisition session. All of the PS rats $(\mathrm{n}=$ 8) acquired the lever-press response, whereas only a few of the CON rats $(n=3)$ acquired the response. This replicates and extends the findings of previous aversive conditioning tasks conducted with PS rats that exposure to prenatal stressors result in facilitated learning in offspring (e.g., Dickerson et al., 2005; Griffin et al., 2003; Ward et al., 2000). This study demonstrates that facilitated learning in PS rats also extends to a positive reinforcement task.

The difference seen in response acquisition between PS and CON rats may not be solely attributed to differences in locomotor activity between the two groups, because there was no statistical difference between groups with response rates on the inoperative lever. Also, for those rats that acquired the response, there was no difference between groups on the number of lever presses on either lever during the delay. If differences in acquisition were due to greater general levels of activity in PS rats, the PS rats may have also emitted higher rates on the inoperative lever, or had a greater number of lever presses during the delay to food pellet delivery, compared to $\mathrm{CON}$ rats. Instead, the differences found between the two groups with responding on the operative lever during acquisition may be attributed to prenatal exposure to acute stressors.

Prior research has demonstrated that the prenatal stress protocol used in the present set of experiments (i.e., exposure of the dam to a novel environment and saline injection daily) resulted in offspring being exposed to high plasma levels of endogenous glucocorticoids (Dickerson et al., 2005). This results in a hyperactive HPA axis in adult PS rats, as is reflected in higher levels 
of serum corticosterone following application of stressors. The serotogenic and noradregenic neurotransmitter systems are also altered in PS rats, as is evident by the greater plasma levels of norepinephrine following stressors than control rats. Behavioral differences between PS and $\mathrm{CON}$ rats have been found as well, including enhanced conditioned fear and increased behavioral inhibition in response to footshock, increased defensive withdrawal in response to restraint, as well as increased freezing and less exploration in aversive environments such as open fields and elevated mazes (Dickerson et al., 2005; Griffin et al., 2003; Ward et al., 2000). Together, these studies suggest that exposure to prenatal stress may enhance the association between aversive stimuli and elicited behavior (e.g., freezing, defecating) during aversive conditioning tasks. Experiment 1 demonstrated that exposure to prenatal stressors facilitated learning in offspring using an operant task. Previous operant research conducted with PS rodents found that prenatal stress resulted in learning deficits during spatial learning tasks relative to CON rodents (Vallee et al., 1999; Kapoor et al., 2009).

There are conflicting results in the literature as to whether prenatal stress impedes or facilitates learning in offspring. Studies conducted by Vallee et al. (1999) and Kapoor et al. (2009) found that rodents exposed to prenatal stress display learning impairments during spatial learning tasks. This discrepancy in findings may be due to procedural or subject variables. The prenatal stressor used by Vallee et al. was restraining the dams for three 45-min periods per day. The authors also used a different procedure to assess learning (i.e., number of visits to a novel arm of a Y-maze and number of errors made in a radial arm maze). Kapoor et al. examined guinea pigs (the current study was conducted with rats), and used exposure to a high frequency strobe light for $2 \mathrm{~h}$ on three consecutive days during pregnancy (i.e., gestational days 50, 51, and 52 , or gestational days 60,61 , and 62 ). Kapoor et al. measured learning by measuring the 
distance covered before finding a hidden platform in a water maze. More research is needed to determine effects of different procedural variables (e.g., species, learning task, type of prenatal stress) on learning.

As of yet, it is unclear as to whether the behavioral differences found between PS and CON rats serve adaptive or maladaptive functions. PS rats have been shown to exhibit increased fear when exposed to a novel open field (i.e., greater latencies to enter open field and fewer exits from enclosed chamber) relative to CON rats (Dickerson et al., 2005). In addition, PS rats have displayed increased freezing when placed in an environment that had been previously paired with shock (Griffin et al., 2003). In the case of enhanced fear responses to aversive stimuli, this increased freezing and decreased exploration may be viewed as serving an adaptive function. These fear responses may be important to survival, as remaining motionless in a novel environment for extended periods of time may help to avoid detection by a predator. However, PS guinea pigs take longer to find a hidden platform in a water maze (Kapoor et al., 2009), PS monkeys exhibit greater social avoidance following social isolation (Schneider et al., 2002; Schneider, 1992; Clarke \& Schneider, 1993), and PS rats exhibit impaired spatial learning in maze tasks compared to CON rats (Vallee et al., 1999). In these studies, the altered behaviors may be viewed as maladaptive because they adversely impact the likelihood of death from drowning, impede the nurturing of social relationships which may be important for survival, and adversely affect efficient exploration of the environment, respectively. Ultimately, whether behavioral differences between PS and CON animals serve an adaptive or maladaptive function may be determined by the context of the situation.

A final note should be made regarding the weight differences found between litters. Rats from the first litter (litter A) weighed significantly more than rats in the second litter (litter B). 
This difference was only about $30 \mathrm{~g}$, and was probably due to the rats in litter A beginning Experiment 1 at a slightly older age than the rats from litter B (i.e., the rats from the first litter were closer to 70 days old, and the rats from the second litter were closer to 60 days old). However, this did not affect acquisition of the lever-press response, as no significant differences were found between litters with response rates on the operative lever or food pellets obtained during the acquisition session.

\section{Experiment 2}

There is a well established correlation between higher rates of delay discounting (impulsive choice) and increased drug self-administration in the non-human animal literature (Setlow et al., 2009; Perry et al., 2005; Logue et al., 1998). Human drug abusers exhibit greater rates of delay discounting compared to non-drug-using control participants (Field et al., 2007; Mitchell et al., 2005; Odum et al., 2002). There has also been a link found between increased physiological indicators of stress and increased acquisition and maintenance of drug-self administration (West \& Weiss, 2006; Sinha, 2001; Mantsch et al., 1998; Taylor et al., 1990). Thus, there may be a link between increased stress and increased rates of delay discounting. There has been very little work conducted investigating the impact of increased stress on impulsive behavior, but a correlation has been found between increased levels of stress and higher rates of delay discounting in humans (Fields et al., 2009; White et al., 2009). The purpose of Experiment 2 was to examine effects of prenatal stress on delay discounting in rats.

\section{Method}

\section{Subjects \& Apparatus}

The eight PS male rats and eight CON male that served as subjects in Experiment 1 served as subjects in Experiment 2. Subjects were approximately three months old at the start of 
Experiment 2. Subjects were housed in similar conditions and were exposed to the same food and water regimens as described in Experiment 1. The same eight operant-conditioning chambers used in Experiment 1 were used in Experiment 2.

\section{Initial Training}

Before subjects were exposed to the delay-discounting procedure, initial training was implemented to ensure that all subjects were responding reliably. Initial lever-press training for all subjects included both levers. Food was delivered according to a conjoint FR 1 VT 60-s schedule. Values for the VT were obtained using a Fleshler-Hoffman sequence generator for 20 cycles of reinforcer delivery. One food pellet was delivered after a lever press on either lever or after an average of $60 \mathrm{~s}$ had elapsed. When most food pellets were obtained via the lever press (i.e., at least 35 out of 40 pellets), an FR 1 schedule alternated between the left and right levers after the delivery of five food pellets on each lever for 40 food pellets total. If lever pressing was not reliably maintained, it was shaped by reinforcing successive approximations.

\section{Delay-Discounting Procedure}

Once food pellets were reliably earned for responses on both levers, the choice procedure began. This procedure was based upon that first used by Evenden and Ryan (1996) for examining impulsive choice in rats. Sessions consisted of five blocks of eight trials each, in which each block was comprised of two forced-choice followed by six free-choice trials. Trials began every $100 \mathrm{~s}$. Forced-choice trials ensured exposure to both sets of contingencies before allowing a choice between them. Forced-choice trials began with one lever, randomly determined, extended into the chamber and the light above that lever illuminated. The lever associated with the larger reinforcer (three food pellets) was counterbalanced between subjects within each group. If a response was emitted on the lever associated with the smaller, immediate 
reinforcer, the lever was retracted, all lights in the chamber were extinguished (i.e., blackout period), and one food pellet was delivered immediately. If a response was emitted on the lever associated with the delayed outcome, the lever was retracted, and the lever light was extinguished. The houselight remained illuminated during the delay. Following the delay, the houselight was extinguished (i.e., blackout period) and three food pellets were delivered. Since the duration of time between the start of each trial was held constant across all trials and blocks (i.e., 100 s), choice for the smaller reinforcer to the exclusion of the larger reinforcer resulted in loss of overall reinforcement, and thus may be considered to be maladaptive. After the blackout period had elapsed, the second forced-choice trial began. The houselight was illuminated, the other lever was extended into the chamber, and the light above the lever was illuminated. A response upon the lever resulted in either the immediate or delayed consequence (whichever one had not been presented during the previous forced-choice trial), as previously described. A 30-s limited hold was in effect for all trials. Failure to respond within $30 \mathrm{~s}$ of the onset of the trial resulted in retraction of the response lever, extinguishing of the houselight and lever lights, and the trial was recorded as an omission.

After exposure to both the immediate and delayed consequences in the two forced-choice trials, six free-choice trials began. At the beginning of each free-choice trial, both levers were extended into the chamber and the houselight and lights above both levers were illuminated. The subjects were then allowed to choose one alternative. Once a choice was made, both levers were retracted and both lever lights were extinguished. The immediate and delayed choice consequences were the same as in the forced-choice trials. Failure to respond within $30 \mathrm{~s}$ of the onset of the trial resulted in retraction of both levers, the houselight and lever lights were extinguished, and the trial was recorded as an omission. Following completion of the six free- 
choice trials within a block, the delay to the larger reinforcer was increased and presented in the forced- and free-choice trials in the next block. The smaller reinforcer (one food pellet) was always presented immediately. In the first block, the value of the delay to the larger reinforcer was always $0 \mathrm{~s}$. The value was then increased across blocks in ascending order. Initially, the values were increased in the order of $0,1,2,4,6 \mathrm{~s}$. The terminal value of the delay to the larger reinforcer was increased gradually over sessions (cf. Anderson \& Woolverton, 2005). Once the sessions were reliably completed and choice for the larger reinforcer in the first (0-s) block was $80 \%$ or greater for three consecutive sessions, the delays were increased to $0,2,4,8,16 \mathrm{~s}$. Using the same criteria, the delays were increased again to $0,5,10,20,40 \mathrm{~s}$ and finally to $0,10,20,40$, 60 s. As delays were increased within a session, it was expected that choice would shift from the larger reinforcer (self-control choice) to the smaller reinforcer (impulsive choice). For individual subjects, the terminal delay value was decreased if a floor effect was observed, and increased if a ceiling effect was observed. This resulted in intermediate delay-discounting functions. For some subjects, choice was almost exclusively for the larger reinforcer, even at the longest delay sequence $(0,10,20,40,60 \mathrm{~s})$. To rule out a possible lever bias, the lever associated with the larger reinforcer was reversed. The procedure for increasing the delay value to the larger reinforcer across sessions was then repeated.

Sessions terminated following 40 trials (10 forced-choice and 30 free-choice) and lasted 66.67 minutes. Experimental sessions were conducted on average six days per week. Each delay series was in effect for at least five sessions and until responding was stable. Stability criteria were identical to that required for increasing the terminal delay value, with the following exceptions. There was a minimum of 20 sessions conducted at the terminal delay value. No more than $20 \%$ variation between numbers of larger reinforcer choices in each block and no evidence 
of an increasing or decreasing trend in the last five sessions at the terminal delay value was to be observed. On Wednesdays of each week, the delay to the larger reinforcer was set to $0 \mathrm{~s}$ across all blocks. These probe sessions were conducted to ensure that responding was sensitive to food pellet amount (one vs. three food pellets). Choice was expected to be maintained by the larger reinforcer option across all blocks. If preference was for the larger reinforcer (i.e., at least five out of six free-choice trials in each block were for the larger reinforcer), the current delay value series were reinstated the next day. However, if choice was not maintained by the larger reinforcer (i.e., less than five free-choice trials in each block were for the larger reinforcer), probe sessions were continued until this criterion was met.

\section{Data Analysis}

Data were reported as mean percent choice for the larger reinforcer as a function of increasing delay values. This is consistent with other published studies (cf. Evenden \& Ryan, 1996; 1999; Anderson \& Woolverton, 2005; Cardinal, Pennicott, Sugathapala, Robbins, \& Everitt, 2001; Cardinal, Robbins, \& Everitt, 2000). An indifference point (s) was interpolated by determining at which delay choice between the smaller and larger reinforcer was equal. Indifference points (s) were interpolated by fitting a logistic equation by non-linear regression. By comparing delay-discounting functions and indifference points, conclusions may be drawn regarding effects of prenatal stress on impulsive choice. Statistical analyses of the data were used to determine significant differences between groups on measures of body weight, sessions required to pass 0 -s probes, sessions required before stability, and average indifference points from the last five sessions, (One-Way Analysis of Variance; ANOVA). 


\section{Results}

At the start of Experiment 2, the PS rats weighed an average of $275.0 \mathrm{~g}(S E M=5.63 \mathrm{~g})$, and the CON rats weighed an average of $265.50 \mathrm{~g}(S E M=4.40 \mathrm{~g})$. This difference in weight was not statistically significant $[f(1,13)=1.87, \mathrm{p}=0.195]$. There were no differences between litters A and B in weight or any of the dependent measures used in Experiment 2, and thus, data from the two litters (PSa \& PSb and CONa \& $\mathrm{CONb}$ ) were combined. The data from PSb-2 were discarded due to a pervasive lever bias, and thus excluded from the analyses.

Figure 4 (top panel) shows group summary data of percent choice for the larger reinforcer as the delay to its delivery increased across delay blocks during the last five sessions. As the delay to the larger, delayed reinforcer increased, choice for that alternative decreased (i.e., delay discounting was observed in all subjects). Group mean indifference points are presented in Figure 4 (bottom panel). Although the PS rats had a group mean indifference point $(M=18.27$ seconds, $S E M=7.21$ seconds $)$ more than twice that of the CON rats $(M=7.79$ seconds, $S E M=$ 1.21 seconds), this difference was not statistically significant, $[f(1,14)=2.35, \mathrm{p}=0.149)$. The range of mean indifference points was much wider for the PS rats (range $=3.0-49.6 \mathrm{~s}$ ) than the $\mathrm{CON}$ rats (range $=3.17 \mathrm{~s}-14.0 \mathrm{~s})$. 

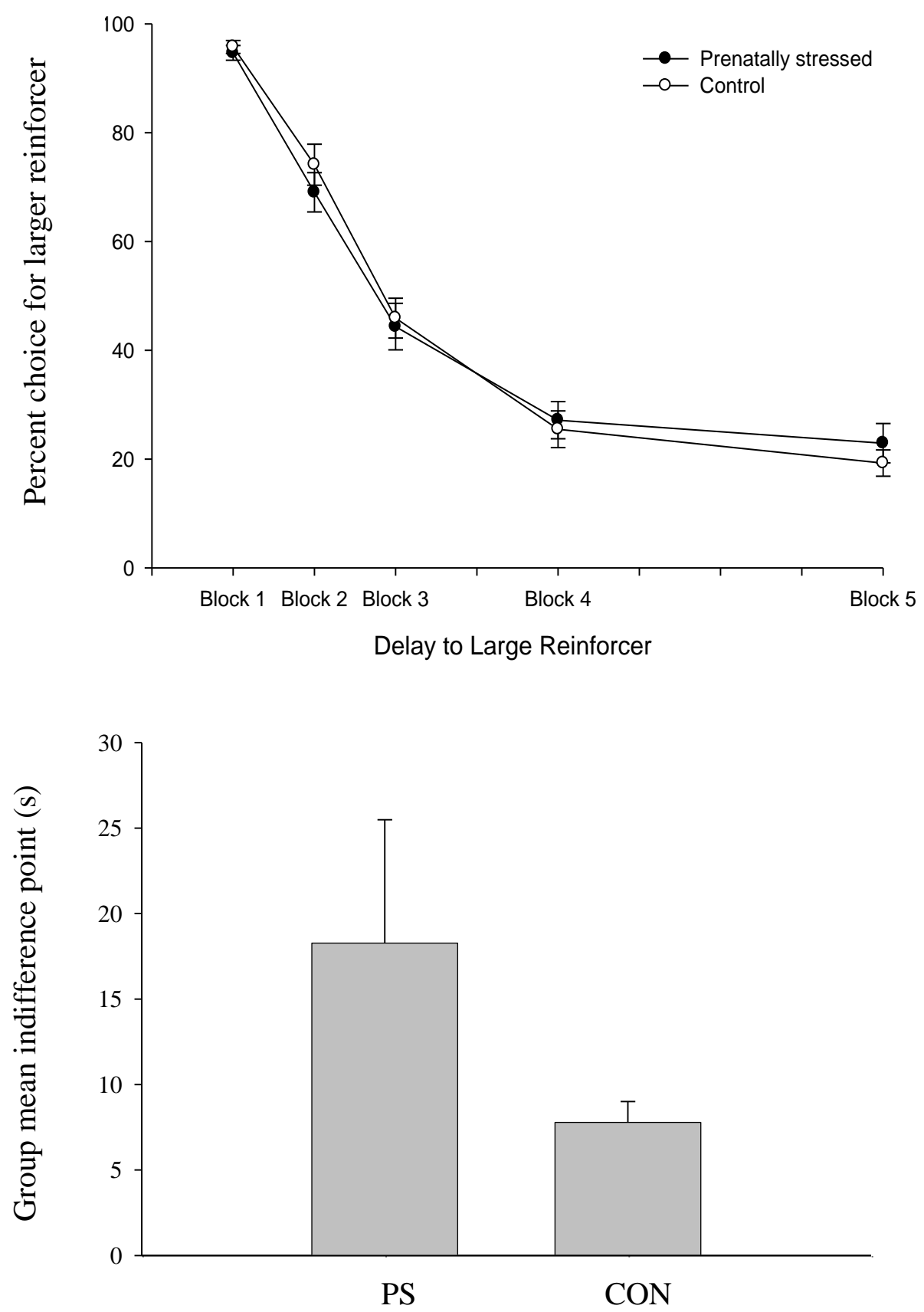

Figure 4. Group mean percent choice for the larger reinforcer as a function of delay block (top panel) and group mean indifference points (bottom panel) for PS and CON rats for the last five sessions during Experiment 2. SEM for all data are presented. 
Table 1 shows terminal delay values and indifference points for individual subjects.

Intermediate delay-discounting functions were obtained for each subject (i.e., terminal delay values were adjusted until no floor or ceiling effects were observed).

Table 1. Indifference point (s) and terminal delay value for individual subjects.

\begin{tabular}{|c|c|c|}
\hline Subject ID & Terminal Delay (s) & Indifference Point (s) \\
\hline PSa-1 & 60 & 9.52 \\
\hline PSa-2 & 60 & 49.60 \\
\hline PSa-3 & 60 & 11.74 \\
\hline PSb-1 & 60 & 41.84 \\
\hline $\mathrm{PSb}-3$ & 16 & 6.34 \\
\hline $\mathrm{PSb}-4$ & 16 & 3.00 \\
\hline PSb-5 & 16 & 5.84 \\
\hline \multicolumn{2}{|c|}{ PS mean indifference point (SEM) } & $18.27 \mathrm{~s}(7.21 \mathrm{~s})$ \\
\hline CONa-1 & 16 & 5.80 \\
\hline $\mathrm{CONa}-2$ & 60 & 14.00 \\
\hline $\mathrm{CONa}-3$ & 6 & 3.17 \\
\hline $\mathrm{CONa}-4$ & 40 & 7.90 \\
\hline $\mathrm{CONa}-5$ & 16 & 4.80 \\
\hline CONa-6 & 60 & 10.20 \\
\hline $\mathrm{CONb}-1$ & 16 & 7.04 \\
\hline $\mathrm{CONb}-2$ & 40 & 9.42 \\
\hline CON mean & erence point (SEM) & $7.79 \mathrm{~s}(1.21 \mathrm{~s})$ \\
\hline
\end{tabular}


On Wednesdays, the delay to the larger reinforcer was set to $0 \mathrm{~s}$ across all blocks to ensure that responding was sensitive to food pellet amount (one vs. three food pellets). These probe sessions were repeated until choice was maintained by the larger reinforcer (i.e., five or six free-choice trials in each block were for the larger reinforcer). The PS rats required an average of 1.71 days $(S E M=0.16$ days $)$ and the $C O N$ rats required an average of 2.52 days $(S E M=0.47$ days) each week to meet this criterion. This difference between groups was not statistically significant, $[f(1,14)=2.41, \mathrm{p}=0.145]$.

For data to be considered to be stable, a minimum of 20 sessions were conducted at the terminal delay value, and there was to be no more than $20 \%$ variation between numbers of larger reinforcer choices in each block across sessions. Also, no evidence of an increasing or decreasing trend in the last five sessions of a condition was to be observed. Subjects in the CON group took slightly longer to reach stability $(M=33.38$ sessions, $S E M=3.10$ sessions $)$ than subjects in the PS group $(M=26.71$ sessions, $S E M=3.56$ sessions $)$. This difference between groups was not statistically significant, $[f(1,14)=2.01, \mathrm{p}=0.180]$.

\section{Discussion}

Delay discounting was observed in all subjects (i.e., as the delay to delivery of the larger reinforcer increased, percent choice for that alternative decreased). There were no differences found between PS and CON groups with indifference points, sessions until stability, or average number of sessions per week needed to pass 0 -s probes. There were also no differences in body weight found between PS and CON rats. In addition, no differences in body weight or any of the dependent variables used in Experiment 2 were observed between rat litters, which allowed for the data to be collapsed across groups. 
At this point in time, it is unclear why exposure to prenatal stress did not result in disparate rates of delay discounting relative to $\mathrm{CON}$ rats. There has been very little published work conducted investigating the impact of stress on impulsive behavior. White et al. (2009) found that human participants with a particular genotype associated with decreased striatal binding of D2 receptors showed increased measures of impulsivity on a computerized twochoice impulsivity paradigm (TCIP). Fields et al. (2009) suggested that increased rates of delay discounting may mediate the relation between stress and tobacco use in adolescents (i.e., adolescent smokers reported higher levels of stress and had higher rates of delay discounting compared to non-smoking peers). The current study was the first to investigate effects of prenatal exposure to an acute stressor on delay discounting. It was also the first to examine the link between prenatal stress and delay discounting using non-human animals.

The lack of significant findings in the present study may be due to species or procedural differences between the present study and the studies conducted by White et al. and Fields et al. The present experiment employed rats as subjects, and the other two studies both used human participants. In order to establish the operant response used as a measure in the present procedure, subjects were food restricted for approximately $22 \mathrm{~h}$ per day. The current contingency (i.e., hungry rats working for food) may have overwhelmed effects of a distal stressor experienced in utero. The prevailing current environmental conditions may have overridden the preexisting physiological changes that occurred following exposure to prenatal stress. Investigating the use of a reinforcer that does not require a state of deprivation (e.g., electrical brain stimulation) on delay discounting in PS rats may be beneficial. Also, the participants in the White et al. and Fields et al. studies were either exposed to an acute stressor or reported being stressed at the time of the experiment. Perhaps if the subjects in the current study were exposed 
to acute stressors immediately before experimental sessions, differences in delay discounting may have been observed between PS and CON rats. Physiological indicators of stress were not assessed during this experiment. The use of such a measure (e.g., measuring plasma corticosterone and ACTH levels) would be useful to assess differences in levels of serum corticosteroids between groups.

It is also possible that the measure of impulsivity used in the current study was not sensitive enough to detect differences between groups. The construct of impulsivity is conceptually and operationally multifaceted (Meda et al., 2009). There are different methods of assessing the construct of impulsivity, with delay discounting being just one of them. Another measure of impulsivity that may be more sensitive is one measuring behavioral inhibition. It has been suggested that behavioral inhibition is the most salient characteristic of ADHD (Barkley, 1997). Behavioral inhibition may be assessed by performance on tasks requiring withholding of an operant response, delaying a response, ceasing ongoing responding, or resisting distraction or disruption by competing stimuli (Barkley, 1997). For example, an alternative procedure that may have been utilized is the signaled nosepoke task (cf. Logue et al., 1998). In such a procedure, nosepokes into a hole in the operant chamber are reinforced with food pellets. Once food pellets are reliably being earned, food is made available after nosepoke responses only in the presence of an auditory stimulus (e.g., a tone). Efficiency during the nosepoke task is defined as the ability to withhold a nosepoke response until the auditory stimulus signals that reinforcement is available. This efficiency is considered to be a measure of impulsivity. Future work investigating effects of prenatal stress on impulsivity may involve other operational definitions of impulsivity, (e.g., ability to withhold a nosepoke response). 
Although no link between prenatal stress and increased levels of delay discounting was found in the current experiment, it is possible that such a link does exist. Previous research conducted with human participants has indicated a correlation between heightened stress and greater measures of impulsivity (White et al., 2009; Fields et al., 2009). The use of an acute stressor prior to delay discounting sessions, or implementing a different measure of impulsivity, may yield significant findings. More research is needed to investigate a possible link between greater impulsivity and increased physiological indicators of stress.

\section{Experiment 3}

The purpose of Experiment 3 was to examine effects of prenatal stress on ethanol selfadministration in rats. Research conducted with humans has found that acute and chronic stress plays an important role in increasing vulnerability to drug abuse (Sinha, 2001). Experimental research conducted with non-human animals has found a link between indicators of stress and drug self-administration (Mantsch et al., 1998; West \& Weiss, 2006; Taylor et al., 1990; Piazza et al., 1990; Goeders \& Guerin, 1994; Schenk et al., 1987). Some studies have found that application of prenatal stress in particular leads to increased drug self-administration of psychostimulants (Kippin et al., 2008; Deminiere et al., 1992; Henry et al., 1995; Thomas et al., 2009). There has been little work conducted investing effects of prenatal stress on ethanol selfadministration. DeTurck \& Pohorecky (1987) found that prenatally stressed rats showed attenuated responses to acute ethanol (i.e., decreases in body temperature, startle amplitude, and motor coordination) relative to CON rats. Darnaudery et al. (2007) found that exposing alcoholpreferring female rats to intense stressors (i.e., shock) reduced ethanol self-administration in rats that had not been exposed to prenatal stress. However, ethanol self-administration did not decrease in alcohol-preferring rats that had been exposed to prenatal stress. Experiment 3 of the 
present set of studies examined whether PS male rats would self-administer more ethanol than CON rats in the absence of any acute stressor.

\section{Subjects \& Apparatus}

The eight PS male rats and eight CON male that served as subjects in Experiments 1 and 2 served as subjects in Experiment 3. Subjects were approximately seven to eight months old at the start of Experiment 3. Housing conditions were similar to those used in Experiments 1 and 2. During Experiment 3, subjects were water restricted and given free access to food. Subjects were given $30 \mathrm{~min}$ free access to water $30 \mathrm{~min}$ after the completion of each ethanol self-administration session. This schedule resulted in approximately 22 hours of liquid deprivation prior to the start of each session. The liquid restriction regimen was in accordance with NIH and Institutional Guidelines and was approved by the West Virginia University Animal Care and Use Committee.

During Experiment 3, sessions were conducted in individual subject homecages $(35.5 \times 26.7 \times 22.9 \mathrm{~cm})$ which were placed in an experimental room. The cages were made of Plexiglas and had a metal wire lid. The metal lid contained a centered food pellet reservoir (11.4 $\times 12.7 \times 7.6 \mathrm{~cm}$ ) with two water bottle reservoirs on either side. Each water bottle reservoir was $7.6 \mathrm{~cm}$ wide, $22.9 \mathrm{~cm}$ deep, and protruded $5.1 \mathrm{~cm}$ into the cage on one end and $7.6 \mathrm{~cm}$ on the other end. White noise projected from a speaker served to mask extraneous noise. Two water bottles holding either water or an ethanol solution were placed in the bottle reservoirs on either side of the food receptacle for the duration of the session.

\section{Sucrose Fading}

In order to initiate ethanol self-administration, a standard sucrose-fading procedure was used (cf. Samson, Files, \& Brice, 1996; Samson, Sharpe, \& Denning, 1999; Thanos et al., 2001). Sessions were conducted for one hour a day, seven days per week. Initially, choice was between 
water and $20 \%(\mathrm{w} / \mathrm{v})$ sucrose solution for two sessions. The sucrose solution was reduced to $10 \%$ (w/v) for a minimum of ten sessions, and until $75 \%$ of the daily session intake was from the sucrose solution for five consecutive sessions (or a maximum of 20 sessions). This was to ensure that choice was maintained by the sucrose solution over water. Ethanol was then added to the sucrose solution in graded amounts, and the sucrose concentration was subsequently faded out. The minimum number of sessions at each concentration is consistent with past studies (e.g., Thanos et al., 2001; Tolliver, Sadeghi, \& Samson, 1988). Subjects also had to be reliably drinking the solution (defined by at least $75 \%$ of total session fluid intake for the last five sessions at a given concentration) to progress to the next concentration. Because some subjects were not meeting the criterion of $75 \%$ of total fluid consumed from the ethanol/sucrose bottle, following the concentration of sucrose $10 \%$, ethanol $4 \%$, the criterion was lowered to $20 \%$ of total fluid consumed from the ethanol solution bottle. Refer to Table 2 for the sequence of concentrations, and the minimum (and maximum if applicable) number of sessions at each step. 
Table 2. Minimum number of sessions at each successive concentration of sucrose and ethanol solution. Maximum number of sessions are presented in parentheses if applicable.

Concentrations are expressed as percent of solution.

\begin{tabular}{|c|c|c|}
\hline $\begin{array}{l}\text { Sucrose concentration } \\
(\% \mathrm{w} / \mathrm{v})\end{array}$ & $\begin{array}{c}\text { Ethanol concentration } \\
(\% \mathrm{v} / \mathrm{v})\end{array}$ & $\begin{array}{l}\text { Minimum (maximum) } \\
\text { sessions }\end{array}$ \\
\hline 10 & 0 & $10(20)$ \\
\hline 10 & 1 & $6(20)$ \\
\hline 10 & 2 & $6(17)$ \\
\hline 10 & 4 & $6(9)$ \\
\hline 10 & 7 & 6 \\
\hline 10 & 10 & 6 \\
\hline 10 & 12 & 6 \\
\hline 10 & 14 & 6 \\
\hline 10 & 17 & 6 \\
\hline 10 & 20 & 6 \\
\hline 7 & 20 & 6 \\
\hline 5 & 20 & 6 \\
\hline 4 & 20 & 6 \\
\hline 2 & 20 & 10 \\
\hline 1 & 20 & 10 \\
\hline 0 & 20 & 10 \\
\hline
\end{tabular}


The position of the ethanol solution and water bottles were alternated daily in order to minimize a side bias (LRLRX, with $\mathrm{X}$ being determined by a coin flip). Upon completion of the 1-hr session, the ethanol solution and water bottles were removed. Homecages were removed from the experimental room to the vivarium. Free access to water was given for $30 \mathrm{~min}$ in the vivarium approximately $30 \mathrm{~min}$ after the session was completed.

\section{Two-Bottle Choice Test}

Following completion of the sucrose-fading procedure, the two-bottle choice assessment began. Choice was between tap water and ethanol (10, 15, and 20\% vol/vol). Sessions lasted $1 \mathrm{hr}$ and were conducted seven days per week. The position of the ethanol solution and water bottles were alternated daily, and the order of ethanol concentration presentation was counterbalanced within groups. Each concentration was presented at least twice in each position (four times total). Data collection was identical to that used during sucrose fading. Additional sessions were conducted if sufficient variability around the mean was observed via visual analysis of the data.

\section{Data Analysis}

For all stages, amount of fluid consumed (mls) from each bottle was recorded, and percent of total fluid intake from the ethanol solution and $\mathrm{g} / \mathrm{kg}$ ethanol consumed were calculated. During sucrose fading and testing, data were analyzed from the last five sessions at each successive concentration. A Repeated Measures Analysis of Variance (ANOVA) was conducted to assess differences in ethanol consumption $(\mathrm{g} / \mathrm{kg})$ across PS and CON groups during the sucrose-fading procedure and two-bottle choice test. During the two-bottle choice test, an ANOVA was conducted to assess differences in ethanol consumption (mls and percent of total fluid consumed out of the ethanol bottle). Pair-wise comparisons were conducted as follow-up tests in the case that significant interactions were found. SEM was calculated for all data. 
Pearson's correlation coefficient were calculated comparing ethanol consumed $(\mathrm{g} / \mathrm{kg})$ during sucrose fading and the two-bottle choice procedure to indifference points obtained during Experiment 2.

\section{Results}

At the start of Experiment 3, the PS rats weighed an average of $456.71 \mathrm{~g}$ (SEM=11.15 $\mathrm{g})$, and the CON rats weighed an average of $447.00 \mathrm{~g}(S E M=10.46 \mathrm{~g})$. This difference in weight was not statistically significant $[f(1,13)=0.635, \mathrm{p}=0.536]$. There were no differences between litters in regards to weight or any of the dependent measures used in Experiment 3. Therefore data from the two litters (PSa \& PSb and CONa \& $\mathrm{CONb}$ ) were combined. One subject, PSb-5, died shortly after Experiment 3 began. The data from this subject were discarded, and not used in any of the analyses.

Sucrose Fading

During the sucrose-fading procedure, the PS rats on average consumed similar $\mathrm{g} / \mathrm{kg}$ of ethanol $(M=2.29 \mathrm{~g} / \mathrm{kg}, S E M=0.15 \mathrm{~g} / \mathrm{kg})$ to that consumed by CON rats $(M=2.25 \mathrm{~g} / \mathrm{kg}, S E M=$ $0.14 \mathrm{~g} / \mathrm{kg}$ ). The repeated measures ANOVA revealed that there was no main effect of group, $[f$ $(1,13)=0.037, \mathrm{p}=0.850]$. Figure 5 shows the average $\mathrm{g} / \mathrm{kg}$ ethanol consumed for PS and CON rats at each step of the sucrose-fading procedure. As the concentration of ethanol increased, the $\mathrm{g} / \mathrm{kg}$ of ethanol consumed increased for all subjects to a maximum ethanol intake of $3.0-3.5$ $\mathrm{g} / \mathrm{kg}$. The ANOVA revealed that there was a significant main effect of concentration, $[f(1,14)=$ $84.78, \mathrm{p}=0.000]$. There was also a significant group $\times$ concentration interaction, $[f(1,14)=$ $1.81, \mathrm{p}=0.040]$ 


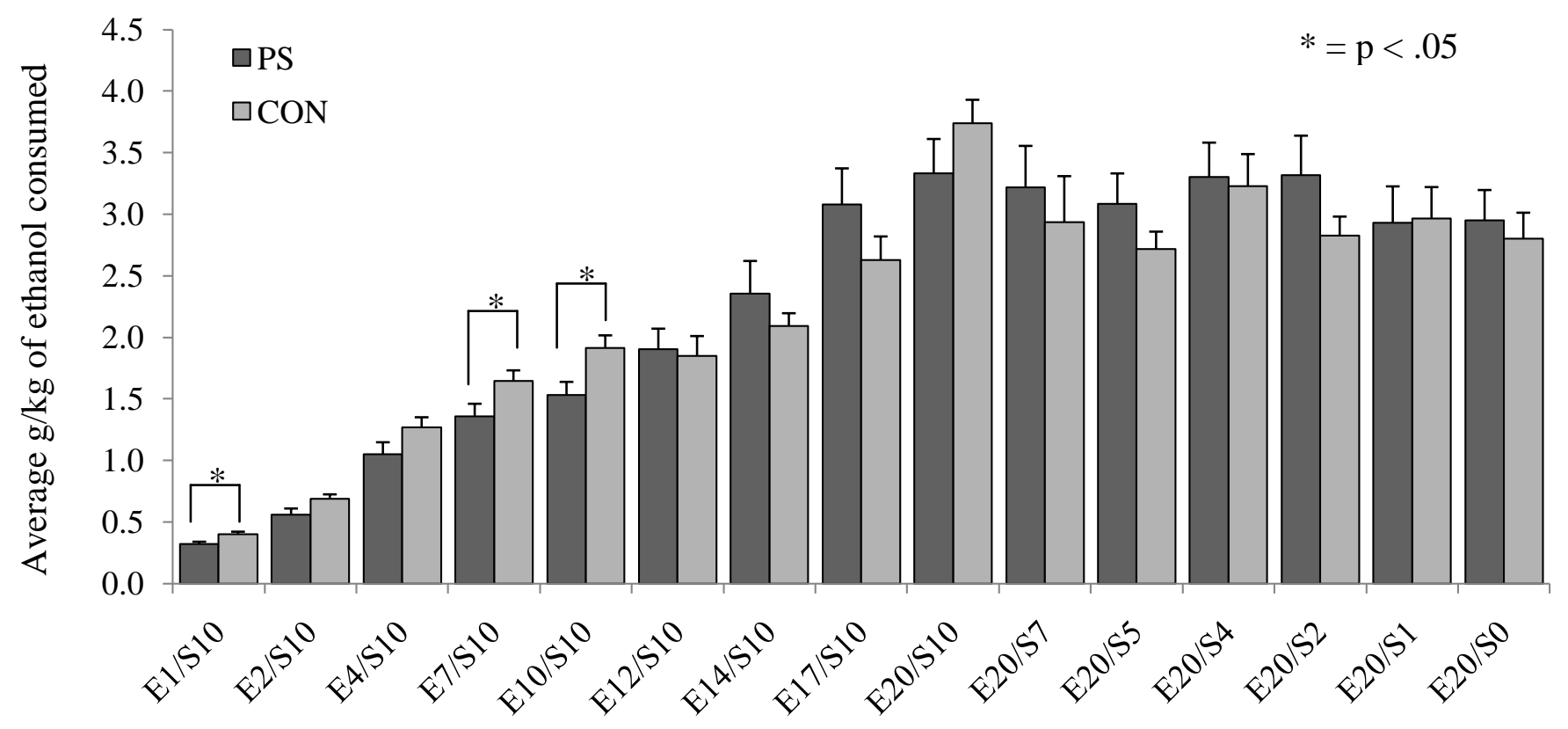

Figure 5. Group means for $\mathrm{g} / \mathrm{kg}$ ethanol consumed at each step during the sucrose fading procedure in Experiment 3 for the last five sessions at each concentration. SEM for all data are presented.

Pair-wise comparisons revealed that at three of the fifteen steps during the fading procedure, the $\mathrm{CON}$ rats on average consumed greater $\mathrm{g} / \mathrm{kg}$ ethanol than the PS rats. The results of these Pair-wise comparisons at concentrations that yielded significant differences are presented in Table 3. 
Table 3. Group mean g/kg of ethanol consumed at concentrations that yielded significant differences as indicated by pair-wise comparisons during the sucrose fading procedure in Experiment 3. SEM, mean difference between groups, and p value at each concentration is also presented.

\begin{tabular}{llllll}
\hline Concentration (\%) & Group & $M$ & SEM & Mean difference & p value \\
\hline Ethanol 1/ Sucrose 10 & PS & 0.319 & 0.020 & 0.800 & 0.020 \\
& CON & 0.399 & 0.022 & & \\
Ethanol 7/ Sucrose 10 & PS & 1.356 & 0.104 & 0.292 & 0.046 \\
& CON & 1.648 & 0.084 & & \\
Ethanol 10/ Sucrose 10 & PS & 1.529 & 0.109 & 0.384 & 0.023 \\
& CON & 1.913 & 0.103 & &
\end{tabular}

Two-Bottle Choice Test

During the two-bottle choice test, PS rats consumed slightly fewer $\mathrm{g} / \mathrm{kg}$ of ethanol $(M=$ $2.18 \mathrm{~g} / \mathrm{kg}, S E M=0.09 \mathrm{~g} / \mathrm{kg})$ than subjects in the CON group, $(M=2.32 \mathrm{~g} / \mathrm{kg}, S E M=0.08 \mathrm{~g} / \mathrm{kg})$. This difference between groups was not statistically significant $[f(1,13)=1.43, \mathrm{p}=0.253]$. As shown in Figure 6 (top panel), as the ethanol concentration increased, the $\mathrm{g} / \mathrm{kg}$ of ethanol consumed increased for all subjects. The ANOVA revealed a significant main effect of ethanol concentration for $\mathrm{g} / \mathrm{kg}$ ethanol consumed, $[f(2,26)=76.035, \mathrm{p}=0.000]$. There was no significant group $\times$ concentration interaction, $[f(2,26)=0.423, \mathrm{p}=0.659]$.

The PS rats drank slightly fewer mls of ethanol solution $(M=9.30 \mathrm{mls}, S E M=0.50 \mathrm{mls})$ than subjects in the CON group, $(M=10.05 \mathrm{mls}, S E M=0.47 \mathrm{mls})$. This difference between groups was not statistically significant $[f(2,13)=1.43, \mathrm{p}=0.253]$. Figure 6 (middle panel) 
shows that the mls of ethanol consumed remained relatively stable across all three testing concentrations. The ANOVA revealed that there was no significant main effect of ethanol concentration for mls of ethanol consumed, $[f(2,26)=0.862, \mathrm{p}=0.43]$. There was also no significant group $\times$ concentration interaction present, $[f(2,26)=0.255, \mathrm{p}=0.777]$.

Subjects in the PS group on average consumed a similar percent of total fluid (intake from ethanol bottle plus water bottle) from the ethanol bottle $(M=47.17$ percent, $S E M=1.68$ percent $)$ as subjects in the CON group, $(M=47.49$ percent, $S E M=1.57$ percent $)$. This difference between groups was not statistically significant $[f(1,13)=0.018, \mathrm{p}=0.894]$. Figure 6 (bottom panel) shows that the percent ethanol of total fluid consumed was similar across all three testing concentrations for both rat groups (range, 45-49\%). The ANOVA revealed that there was no significant main effect of ethanol concentration for percent ethanol consumed, $[f(2,26)=0.859$, $\mathrm{p}=0.435]$. There was also no significant group $\times$ concentration interaction present, $[f(2,26)=$ $0.590, \mathrm{p}=0.562]$. 

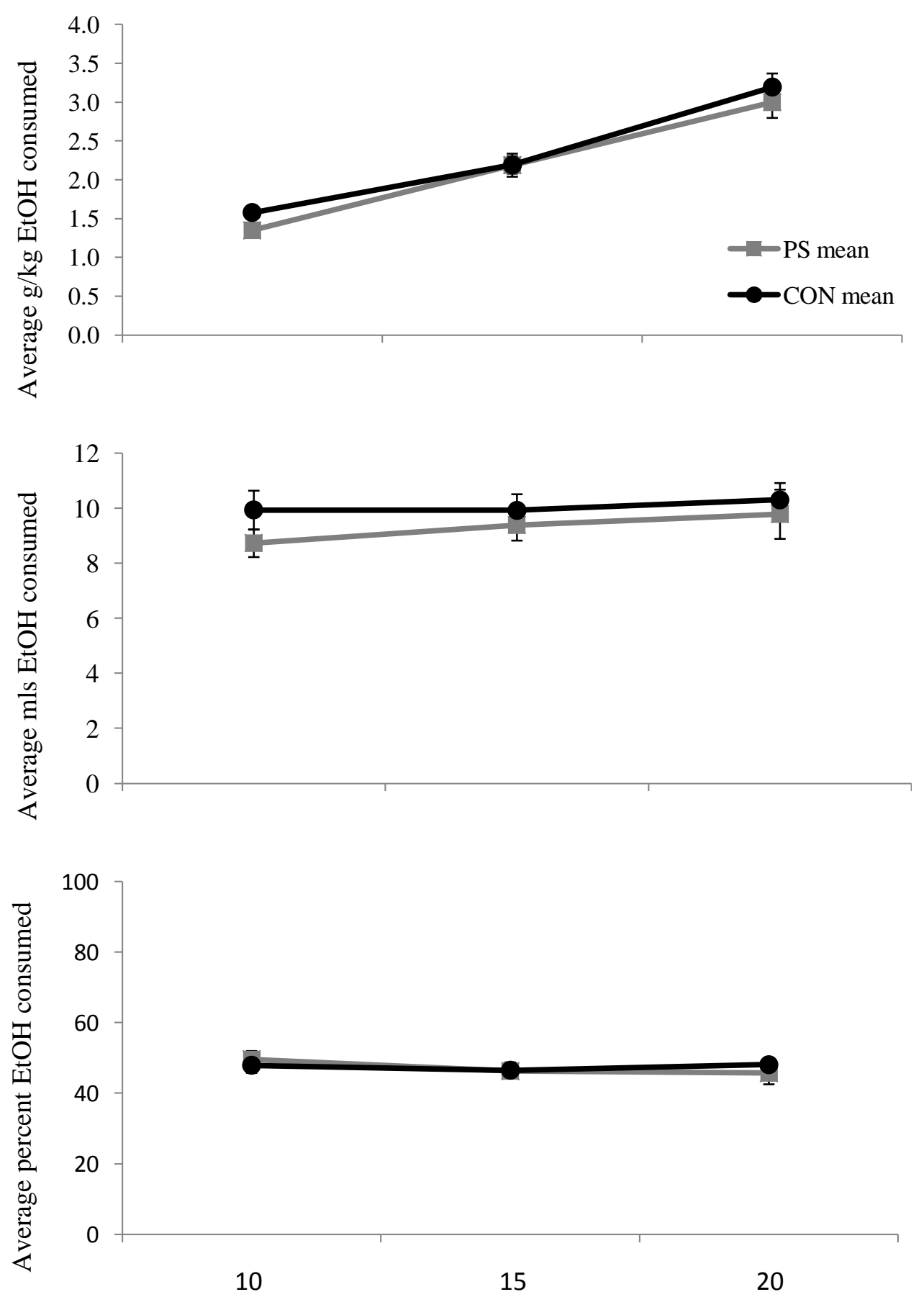

Ethanol (\%) present in solution

Figure 6. Group means for $\mathrm{g} / \mathrm{kg}$ of ethanol (top panel), mls of ethanol (middle panel), and percent ethanol consumed (bottom panel) for each testing concentration during the two-bottle choice test in Experiment 3 for the last five sessions at each concentration. SEM for all data are presented. 
A Pearson correlation indicated that there was a statistically significant negative correlation between obtained indifference points during Experiment 2 and $\mathrm{g} / \mathrm{kg}$ of ethanol consumed during the two-bottle choice test at an ethanol concentration of $10 \%(\mathrm{r}=-0.55, \mathrm{p}<$ 0.05). More specifically, lower indifference points (i.e., greater impulsive choices) were correlated with greater ethanol consumption at the lowest testing concentration. However, the correlations between $\mathrm{g} / \mathrm{kg}$ of ethanol consumed at testing concentrations ethanol $15 \%$ and ethanol $20 \%$ and obtained indifference points were not statistically significant $(r=-0.17, p=$ 0.54 and $r=0.07, p=0.81$, respectively). During the sucrose-fading procedure, a statistically significant negative correlation was found between obtained indifference points and $\mathrm{g} / \mathrm{kg}$ of ethanol consumed at three of the concentrations early in the fading procedure. These were concentrations ethanol $2 \%$, sucrose $10 \%(\mathrm{r}=-0.59, \mathrm{p}<0.05)$, ethanol $4 \%$, sucrose $10 \%(\mathrm{r}=-$ 0.63, $\mathrm{p}<0.05)$, and ethanol $7 \%$, sucrose $10 \%(\mathrm{r}=-0.65, \mathrm{p}<0.01)$. At these three concentrations, greater impulsivity (i.e., lower indifference points) was correlated with greater consumption of ethanol.

\section{Discussion}

During the sucrose-fading procedure, as the ethanol concentration increased, the $\mathrm{g} / \mathrm{kg}$ of ethanol consumed increased for all subjects. Although there was not a main effect of group present, there was a significant group $\mathrm{x}$ concentration interaction for three different concentrations near the beginning of the sucrose-fading procedure (ethanol $1 \%$, sucrose $10 \%$; ethanol 7\%, sucrose 10\%; and ethanol 10\%, sucrose 10\%). At these concentrations, subjects in the CON group consumed significantly more $\mathrm{g} / \mathrm{kg}$ of ethanol than subjects in the PS group. It is important to note that these ethanol concentrations were accompanied by a relatively high concentration of sucrose. It is thus difficult to determine whether the increased consumption by 
CON rats was maintained by the ethanol or the sucrose present in the solution. A group $\mathrm{x}$ concentration interaction was not observed at ethanol concentrations greater than $10 \%$, or after the sucrose concentration was faded out. This suggests that the CON rats consumed greater $\mathrm{g} / \mathrm{kg}$ of ethanol at those three concentrations due to the sucrose present in the solution.

During the two-bottle choice test, the mls of ethanol solution, percent ethanol, and $\mathrm{g} / \mathrm{kg}$ of ethanol consumed did not vary significantly between groups. Choice between ethanol and water was indifferent, as the average percentage of total fluid consumed from the ethanol solution bottle was slightly less than $50 \%$. The amount of ethanol solution consumed (mls) did not vary as a function of concentration. However, when corrected for body weight, there was a main effect of concentration for $\mathrm{g} / \mathrm{kg}$ of ethanol consumed. As the concentration increased, the $\mathrm{g} / \mathrm{kg}$ of ethanol consumed increased for all subjects.

Human substance abusers have been found to discount the value of delayed reinforcers at a greater rate than non-drug-using control participants (Field et al; 2007; Mitchell et al., 2005; Odum et al., 2002). In the non-human animal literature, researchers have consistently found a relation between higher rates of delay discounting and greater drug self-administration (Logue et al., 1998; Perry et al., 2005; Setlow et al., 2009). In the present study, a significant negative correlation was found between obtained indifference points in Experiment 2 and $\mathrm{g} / \mathrm{kg}$ of ethanol consumed at an ethanol concentration of $10 \%$ during the two-bottle choice test in Experiment 3 regardless of group. Subjects with shorter indifference points (i.e., were more impulsive) consumed greater $\mathrm{g} / \mathrm{kg}$ of ethanol at the lowest testing concentration. In addition, a significant negative correlation was found between indifference points and $\mathrm{g} / \mathrm{kg}$ of ethanol consumed at three concentrations early on in the sucrose-fading procedure (ethanol 2\%, sucrose 10\%; ethanol $4 \%$, sucrose $10 \%$; and ethanol 7\%, sucrose 10\%). However, the highest concentration of sucrose 
used was present in the solution of the three concentrations. Therefore, it is difficult to determine whether subjects with lower indifference points (i.e., greater impulsivity) consumed greater $\mathrm{g} / \mathrm{kg}$ of ethanol at the three concentrations because of the ethanol or sucrose present in the solution. These findings are consistent with past research showing greater impulsive behaviors correlating with greater facilitation or maintenance of drug self-administration. Perhaps if concentrations lower than ethanol $10 \%$ were tested, additional negative correlations between obtained indifference points during Experiment 2 and $\mathrm{g} / \mathrm{kg}$ of ethanol consumed during testing may have been found. However, the lowest testing concentration used during the choice test was ethanol $10 \%$, as no differences between groups were found during the sucrose-fading procedure after sucrose was faded out.

There is a well-established link between increased levels of stress and facilitated drug self-administration in both humans and non-human animals. Research has demonstrated that acute and chronic stress plays an important role in the acquisition, maintenance, and relapse of substance abuse in humans (see Sinha, 2001 for a review). Research conducted with non-human animals has yielded similar findings (Piazza et al., 1990; Taylor et al., 1990; Goeders \& Guerin, 1994; Mantsch et al., 1998; West \& Weiss, 2006). Exposure to prenatal stress has been found to result in facilitated acquisition and maintenance of stimulants in rodents (Deminiere et al., 1992; Henry et al., 1995; Kippin et al., 2008; Thomas et al., 2009). PS rats have also shown attenuated responses to acute ethanol (DeTurck \& Pohorecky, 1987), as well as a persistence to selfadminister ethanol following acute shock (Darnaudery et al., 2007) compared to CON rats. It is unclear at this time why no differences between PS and CON rats with ethanol selfadministration were observed in the current study. 
Procedural differences may have attributed to the lack of significant findings in the present study. DeTurck and Pohorecky (1987) and Darnaudery et al. (2007) both used restraint stress as the method of implementing the prenatal stressor; the current study stressed the pregnant dams with exposure to a novel environment and saline injections. Acute stressors were also applied prior to or during the sessions in the previous studies. The subjects in the DeTurck and Pohorecky study were injected with a moderate dose of ethanol. The handling and the injection may have been stressful to the animals. The subjects in the Darnaudery et al. study were exposed to acute footshock prior to ethanol self-administration. Because physiological indicators of stress were not assessed during the present experiment, it is unknown whether the subjects were experiencing heightened levels of stress during these sessions. Previous research have shown PS rats to have a hyperactive HPA axis, as is reflected in higher and more enduring levels of stress hormones in the blood following exposure to stressors (Dickerson et al., 2005). There has been little research conducted investigating whether the offspring of PS rats exhibit heightened HPA axis responses in the absence of an acute stressor. One study by Koehl et al. (1999) found that the daily peak response of corticosterone was higher and occurred sooner in PS rats relative to $\mathrm{CON}$ rats. However, it is unknown whether corticosteroid levels varied between groups during the present experimental sessions.

Subject maturation may also have played an important role in the present findings. The subjects used in the Dickerson et al. study were 25-60 days old, and 3-4 months old in the Koehl et al. study at the start of testing. Subjects in the present study were 7-8 months old at the start of Experiment 3. It is possible that the physiological changes following exposure to the prenatal stressor may not have endured to later adulthood. The use of such a measure (e.g., measuring plasma corticosterone levels) would be useful to assess differences in levels of corticosteroids 
between groups at the time of testing. Examining effects of prenatal stress on ethanol selfadministration following application of an acute stressor such as tail pinch or shock immediately before experimental sessions would also be of interest. More research is needed to investigate effects of prenatal stress on ethanol self-administration.

\section{General Discussion}

\section{Summary of Findings}

During Experiment 1, subjects in the PS group obtained significantly more food pellets and emitted higher response rates on the lever correlated with food (i.e., the operative lever) relative to $\mathrm{CON}$ rats. In addition, visual analysis of the cumulative response graphs indicated that all of the PS rats acquired the lever-press response (all eight subjects), whereas only three of the eight $\mathrm{CON}$ rats acquired the response. This difference in acquisition may not be attributed solely to differences between groups in general locomotor activity, as no differences between groups were observed in response rates on the inoperative lever (i.e., the lever not correlated with food), or absolute number of responses during the delay to food pellet delivery. Had it been due to differences in activity levels, the PS rats may have also had emitted significantly more responses on both levers during the delay to reinforcer delivery, or responded at significantly greater rates on the inoperative lever relative to $\mathrm{CON}$ rats.

The aim of Experiment 2 was to assess effects of prenatal stress on delay discounting. There was no statistically significant difference observed between the PS and CON rats with indifference points during Experiment 2. During Experiment 3, effects of prenatal stress on ethanol self-administration were investigated. At a few concentrations early in the sucrose-fading procedure, the $\mathrm{CON}$ rats consumed significantly greater $\mathrm{g} / \mathrm{kg}$ of ethanol relative to PS rats. These differences were only found at ethanol concentrations lesser than or equal to $10 \%$, and steps at 
which sucrose $10 \%$ was still present in the ethanol solution. Thus, it is difficult to ascertain whether the CON rats consumed greater $\mathrm{g} / \mathrm{kg}$ of ethanol due to the ethanol or the sucrose present in the solution. During the two-bottle choice procedure, in which no sucrose was present in the solution, there were no differences between PS and CON rats for $\mathrm{g} / \mathrm{kg}$ of ethanol consumed at any ethanol concentration.

Previous research has found a link between impulsive behavior and increased facilitation and maintenance of drug taking in both the human and non-human animal literature. Because there were no differences between the PS and CON groups with delay discounting, all discounting data were combined. A significant negative correlation was found between indifference points and $\mathrm{g} / \mathrm{kg}$ of ethanol consumed at a testing concentration of ethanol $10 \%$. More specifically, lower indifference points (i.e., greater impulsive choice) were linked with greater $\mathrm{g} / \mathrm{kg}$ of ethanol consumed. However, no correlations were found at the two higher testing concentrations.

\section{Extension of Literature on Prenatal Stress}

Experiment 1 of the present set of studies served to replicate and extend the findings of previous learning tasks conducted with PS rats that exposure to prenatal stressors may result in facilitated learning in the offspring (Dickerson et al., 2005; Griffin et al., 2003; Ward et al., 2000). This was the first study conducted that demonstrated that exposure to prenatal stress facilitated learning using an operant learning task (i.e., lever-press acquisition). Previous work with aversive conditioning tasks has demonstrated that exposure to prenatal stress enhances the association between aversive stimuli and elicited behavior in rats (Weinstock, 1992; Dickerson et al., 2005; Griffin et al., 2003). However, previous operant research conducted with PS and CON animals has found that exposure to prenatal stress impedes learning (Kapoor et al., 2009; Vallee 
et al., 1999). Thus, there have been mixed findings in the literature regarding whether prenatal stress impedes or facilitates learning. However, interspecies and procedural differences (e.g., prenatal stressor used, operant task used) may have contributed to the discrepant results.

Experiment 2 of the present set of studies was the first to examine effects of prenatal stress on impulsive behavior. There has been very little work conducted examining the link between stress and delay discounting. Because there is a well-established correlation between increased rates of delay discounting and increased drug self-administration, and a link found between increased physiological indicators of stress and increased acquisition and maintenance of drug taking, it is possible that a link may exist between increased stress and delay discounting. The present set of studies was the first to investigate the correlation between these three variables using a within-subject design. This design was unique in that it allowed for correlations between these three variables to be assessed within individual subjects. Although there were no significant differences found between PS and CON groups with delay discounting or ethanol self-administration, a significant correlation was found between performance on the delay discounting task and ethanol self-administration procedure for one of the three testing concentrations. The findings of this experiment that more impulsive choices were correlated with greater drug self-administration are consistent with previous research (e.g., Setlow et al., 2009; Perry et al., 2005; Logue et al., 1998).

\section{Clinical Significance of Findings}

In the clinical literature, prenatal maternal stress in humans has been linked to premature birth, low birth weight, slower development, as well as ADHD and other psychiatric disorders (see Talge et al., 2007 for a review). In the non-human animal literature, results have been mixed. Some studies have found that higher stress in utero may lead to offspring exhibiting 
impaired physical development and attention deficits (Schneider \& Coe, 1993), learning impairments (Kapoor et al., 2009; Vallee et al., 1999), adverse reactions to reunion with peers following social isolation (Schneider et al., 2002; Clarke \& Schneider, 1993; Schneider, 1992), and higher levels of physiological indicators of stress (i.e., cortisol and ACTH) both at baseline and following exposure to acute stressors (Clarke, Wittwer, Abbot, \& Schneider, 1994). Other studies conducted with non-human animals, including Experiment 1 of the present set of studies, have demonstrated that exposure to prenatal stress may result in facilitated learning (Dickerson et al., 2005; Griffin et al., 2003; Ward et al., 2000), both with respondent and operant tasks. It is important to note that these research studies implemented a variety of procedures of inducing stress in the pregnant dams and measuring learning in offspring, which may have contributed to the discrepant findings. In addition, exposure to prenatal stress did not result in detrimental effects during Experiment 2 (impulsive choice) or Experiment 3 (ethanol self-administration). There were no differences observed between groups in regards to these two measures.

Although there have been mixed findings in the non-human animal research, the majority of the clinical research has found that negative outcomes are associated with prenatal maternal stress. However, a few clinical research studies have found that exposure to prenatal stress has no negative outcomes or that it may be beneficial to the offspring. For example, DiPietro et al. (2006) found that moderate levels of maternal stress were associated with optimal early child development. The authors found no association found between maternal stress and deficits in attentional or emotional capacities. Other studies have found no association between maternal report of experiencing anxiety or depression symptoms during pregnancy and low birth weights and shorter gestation periods (Andersson, Sundstrom-Poromaa, Wulff, Astrom, \& Bixo, 2004; Berle et al., 2005). The present set of studies employed a prenatal stressor that was relatively 
mild, which may have contributed to the finding that subjects exposed to prenatal stress exhibited facilitated learning during Experiment 1, and yielded data indistinguishable from CON rats during Experiments 2 and 3. It is also possible that during Experiments 2 and 3, the lack of negative findings may have been due to effects of the distal stressor experienced in utero being washed out by the current contingencies (i.e., food- and water- restricted rats were consuming food and water during experimental sessions). Because a within-subject design was utilized in the present set of studies, the same subjects were used for each experiment. Therefore, subjects had aged significantly from the start of Experiment 1 (aged 2 months) to the start of Experiment 3 (aged 7-8 months). The physiological changes that occurred following exposure to the prenatal stressor may have faded in later adulthood.

\section{Future Directions}

The current study did not employ a physiological measure of stress before or after experimental sessions. However, the procedure of prenatal stress used in the present set of studies is one that is well established (i.e., exposure to a novel environment and injections of saline). Previous research has demonstrated that this procedure exposes developing fetuses to high plasma levels of glucocorticoids, which results in offspring exhibiting altered neurobiology and behaviors persisting into adulthood. PS rats display greater and more enduring levels of corticosterone in the blood following exposure to an acute stressor, which is indicative of a hyperactive HPA axis. There has been little research conducted investigating whether rats show heightened HPA axis responses at baseline. One study (Koehl et al., 1999) found that daily baseline corticosterone levels peak sooner and at greater levels in PS rats relative to CON rats. Schneider (1994) found that PS monkeys had significantly higher cortisol levels at baseline, and higher ACTH levels following exposure to an acute stressor compared to CON monkeys. 
For future studies using PS rats, a physiological measure of glucocorticoids present in the blood may be useful to establish differences between groups at different points in the study. At the present time, it is unknown whether the subjects were experiencing stress during experimental sessions. It is possible that the physiological changes following exposure to prenatal stress were overridden by the present contingencies or physical maturation during the delay discounting and ethanol self-administration procedures. Future studies may also employ an acute stressor prior to sessions. This may assist in establishing differences between groups with delay discounting or ethanol self-administration. It is also possible that the procedure used to assess impulsivity may not have been sensitive enough to detect differences between groups. Future research may investigate effects of prenatal stress using a different measure of impulsivity (e.g., withholding of a nosepoke response). 


\section{References}

American Psychiatric Association (Ed.) (2000). Diagnostic and Statistical Manual of Mental Disorders ( $4^{\text {th }}$ ed. Text Revision). Washington, D.C.: American Psychiatric Association.

Adrover, E., Berger, M.A., Perez, A., Tarazi, F.I., \& Antonelli, M.C. (2007). Effects of prenatal stress on dopamine D2 receptor asymmetry in rat brain. Synapse, 61, 459-462.

Anderson, K.G., \& Elcoro, M. (2007). Response acquisition with delayed reinforcement in Lewis and Fischer 344 rats. Behavioural Processes, 74, 311-318.

Anderson, K.G., \& Woolverton, W.L. (2005). Effects of clomipramine on self-control choice in Lewis and Fischer 344 rats. Pharmacology, Biochemistry, and Behavior, 80(3), 387-93.

Andersson, L., Sundstrom-Poromaa, I., Wulff, M., Astrom, M., \& Bixo, M. (2004). Neonatal outcome following maternal antenatal depression and anxiety: a population-based study. American Journal of Epidemiology, 159, 872-881.

Barkley, R.A. (1997). Behavioral inhibition, sustained attention, and executive functions: constructing a unifying theory of ADHD. Psychological Bulletin, 121, 65-94.

Berle, J.O., Mykletun, A., Daltveit, A.K., Rasmussen, S., Holsten, F., \& Dahl, A.A. (2005). Neonatal outcomes in offspring of women with anxiety and depression during pregnancy. A linkage study from the Nord-Trondelag Health Study (HUNT) and Medical Birth Registry of Norway. Archive of Women's Mental Health, 81, 181-189.

Cardinal, R.N., Pennicott, D.R., Sugathapala, C.L., Robbins, T.W., \& Everitt, B.J. (2001). Impulsive choice induced by lesions of the nucleus accumbens core. Science, 292, 24992501.

Cardinal, R.N., Robbins, T.W., \& Everitt, B.J. (2000). The effects of $d$-amphetamine, chlordiazepoxide, alpha-fluphenthixol and behavioural manipulations on choice of 
signaled and unsignaled delayed reinforcement in rats. Psychopharmacology, 152, 362375.

Clarke, A.S., \& Schneider, M.L. (1993). Prenatal stress has long-term effects on behavioral responses to stress in juvenile rhesus monkeys. Developmental Psychobiology, 26, 293304.

Clarke, A.S., Wittwer, D.J., Abbot, D.H., \& Schneider, M.L. (1994). Long-term effects of prenatal stress on HPA axis activity in juvenile rhesus monkeys. Developmental Psychobiology, 27, 257-269.

Coe, C.L., Kramer, M., Czeh, B., Gould, E., Reeves, A.J., Kirschbaum, C., \& Fuchs, E. (2003). Prenatal stress diminishes neurogenesis in the dentate gyrus of juvenile rhesus monkeys. Biological Psychiatry, 54, 1025-1034.

Darnaudery, M., Louvart, H., Defrance, L., Leonhardt, M., Morley-Fletcher, S., Gruber, S.H., et al. (2007). Impact of an intense stress on ethanol consumption in female rats characterized by their pre-stress preference: modulation by prenatal stress. Brain Research, 1131, 181-186.

Davis, E.P., Snidman, N., Wadhwa, P.D., Glynn, L.M., Schetter, C.D., \& Sandman, C.A. (2004). Prenatal maternal anxiety and depression predict negative behavioral reactivity in infancy. Infancy, 6, 319-331.

Deminiere, J.M., Piazza, P.V., Guegan, G., Abrous, N., Maccari, S., Le Moal, M., et al. (1992). Increased locomotor response to novelty and propensity to intravenous amphetamine selfadministration in adult offspring of stressed mothers. Brain Research, 586, 135-139.

DeTurck, K.H., \& Pohorecky, L.A. (1987). Ethanol sensitivity in rats: effect of prenatal stress. Physiology \& Behavior, 40, 407-410. 
Dickerson, P.A., Lally, B.E., Gunnel, E., Birkle, D.L., \& Salm, A.K. (2005). Early emergence of increased fearful behavior in prenatally stressed rats. Physiology \& Behavior, 86(4), 586593.

DiPietro, J.A., Novak, M.F., Costigan, K.A., Atella, L.D., \& Reusing, S.P. (2006). Maternal psychological distress during pregnancy in relation to child development at age two. Child Development, 77, 573-587.

Evenden, J.L., \& Ryan, C.N. (1996). The pharmacology of impulsive behavior in rats: The effects of drugs on response choice with varying delays of reinforcement. Psychopharmacology, 128. 161-170.

Evenden, J.L., \& Ryan, C.N. (1999). The pharmacology of impulsive behavior in rats VI: The effects of ethanol and selective serotonergic drugs on response choice with varying delays of reinforcement. Psychopharmacology, 146, 413-421.

Field, M., Christiansen, P., Cole, J., \& Goudie, A. (2007). Delay discounting and the alcohol Stroop in heavy drinking adolescents. Addiction, 102(4), 579-586.

Fields, S., Leraas, K., Colins, C., \& Reynolds, B. (2009). Delay discounting as a mediator of the relationship between perceived stress and cigarette smoking status in adolescents. Behavioural Pharmacology, 20, 455-460.

Finlay, J.M., \& Zigmond, M.J. (1997). The effects of stress on central dompaminergic neurons: Possible clinical implications. Neurochemical Research, 22, 1387-1394.

Goeders, N.E., \& Guerin, G.F. (1994). Non-contingent electric footshock facilitates the acquisition of intravenous cocaine self-administration in rats. Psychopharmacology, 114, 63-70. 
Glover, V., O’Connor, T.G., Heron, J., \& Golding, J. (2004). Antenatal maternal anxiety is linked with atypical handedness in the child. Early Human Development, 79, 107-118.

Griffin, W.C., Skinner, H.D., Salm, A.K., \& Birkle, D.L. (2003). Mild prenatal stress in rats is associated with enhanced conditioned fear. Physiology and Behavior, 79, 209-215.

Henry, C., Guegant, G., Cador, M., Arnauld, E., Arsaut, J., LeMoal, M., et al. (1995). Prenatal stress in rats facilitates amphetamine-induced sensitization and induces long-lasting changes in dopamine receptors in the nucleus accumbens. Brain Research, 685, 179-186.

Kapoor, A., Kostaki, A., Janus, C., \& Matthews, S.G. (2009). The effects of prenatal stress on learning in adult offspring is dependent on the timing of the stressor. Behavioural Brain Research, 197, 144-149.

Kippin, T.E., Szumlinski, K.K., Kapasova, Z., Rezner, B., \& See, R.E. (2008). Prenatal stress enhances responsiveness to cocaine. Neuropsychopharmacology, 33, 769-782.

Koehl, M., Darnaudery, M., Dulluc, J., Reeth, O.V., Moal, M.L., \& Maccari, S. (1999). Prenatal stress alters circadian activity of hypothalamo-pituitary-adrenal axis and hippocampal corticosteroid receptors in adult rats of both gender. Journal of Neurobiology, 40, 302315.

Koob, G.F., \& Le Moal, M. (1997). Drug abuse: Hedonic homeostatic dysregulation. Science, $278,252-258$.

Laplante, D.P., Barr, R.G., Brunet, A., Du Fort, G.G., Meaney, M.J., Saucier, J.F., et al. (2004). Stress during pregnancy affects general intellectual and language functioning in human toddlers. Pediatric Research, 56, 400-410.

Lattal, K.A. (2010). Delayed reinforcement of operant behavior. Journal of the Experimental Analysis of Behavior, 93, 129-139. 
Lattal, K.A., \& Gleeson, S. (1990). Response acquisition with delayed reinforcement. Journal of Experimental Psychology: Animal Behavior Processes, 16(1), 27-39.

Logue, S.F., Swartz, R.J., \& Wehner, J.M. (1998). Genetic correlation between performance on an appetitive-signaled nosepoke task and voluntary ethanol consumption. Alcoholism: Clinical and Experimental Research, 22, 1912-1920.

Mantsch, J., Saphier, D., \& Goeders, N. (1998). Corticosterone facilitates the acquisition of cocaine self-administration in rats: opposite effects of the type II glucocorticoid receptor agonist dexamethasone. Journal of Pharmacology and Experimental Therapeutics, 287(1), 72-80.

Mazur, J.E. (1987). An adjusting procedure for studying delayed reinforcement. In M.L. Commons, J.E. Mazur, J.A. Nevin, and H. Rachlin (Eds.), Quantitative analyses of behavior: Vol. 5. The effect of delay and intervening events on reinforcement value. (pp. 55-73). Hillsdale, NJ: Erlbaum.

Meda, S.A., Stevens, M.C., Potenza, M.N., Pittman, B., Gueorguieva, R., Andrews, M.M., et al. (2009). Investigative the behavioral and self-report constructs of impulsivity domains using principal component analysis. Behavioural Pharmacology, 20, 390-399.

Mitchell, J.M., Fields, H.L., D’Esposito, M., \& Boettiger, C.A. (2005). Impulsive responding in alcoholics. Alcohol: Clinical and Experimental Research, 29, 2158-2169.

Niederhofer, H., \& Reiter, A. (2004). Prenatal maternal stress, prenatal fetal movements and perinatal temperament factors influence behavior and school marks at the age of 6 years. Fetal Diagnosis and Therapy, 19, 160-162. 
O’Connor, T.G., Heron, J., Golding, J., Beveridge, M., \& Glover, V. (2002). Maternal antenatal anxiety and children's behavioural/emotional problems at 4 years: Report from the Avon longitudinal study of parents and children. British Journal of Psychiatry, 180, 502-508.

Odum, A.L., Madden, G.J., \& Bickel, W.K. (2002). Discounting of delayed health gains and losses by current, never- and ex-smokers of cigarettes. Nicotine and Tobacco Research, 4, 295-303.

Perry, J., Larson, E., German, J., Madden, G., \& Carroll, M. (2005). Impulsivity (delay discounting) as a predictor of acquisition of IV cocaine self-administration in female rats. Psychopharmacology, 178, 193-201.

Piazza, P.Z., Deminiere, J.M., Le Moal, M., \& Simon, H. (1990). Stress- and pharmacologicallyinduced behavioral sensitization increases vulnerability to acquisition of amphetamine self-administration. Brain Research, 514, 22-24.

Poltyrev, T., Keshet, G.I., Kay, G., \& Weinstock, M. (1996). Role of experimental conditions in determining differences in exploratory behavior of prenatally stressed rats. Developmental Psychobiology, 29, 453-462.

Reynolds, B. (2006). A review of delay-discounting research with humans: relations to drug use and gambling. Behavioural Pharmacology, 17, 651-667.

Rodriguez, A., \& Bohlin, G. (2005). Are maternal smoking and stress during pregnancy related to ADHD symptoms in childhood? Journal of Child Psychology and Psychiatry, 46, 246254.

Rosen, G.D., Finklestein, S., Stoll, A.L., Yutzey, D.A., \& Denenberg, V.H. (1984). Neurochemical asymmetries in the albino rat's cortex, striatum, and nucleus accumbens. Life Sciences, 34, 1143-1148. 
Salm, A., Pavelko, M., Krouse, E.M., Webster, W., Kraszpulski, M., \& Birkle, D. (2004). Lateral amygdaloid nucleus expansion in adult rats is associated with exposure to prenatal stress. Developmental Brain Research, 148, 159-167.

Samson, H., Files, F., \& Brice, G. (1996). Patterns of ethanol consumption in a continuous access situation: The effect of adding a sweetener to the ethanol solution. Alcoholism: Clinical and Experimental Research, 20(1), 101-109.

Samson, H., Sharpe, A., \& Denning, C. (1999). Initiation of ethanol self-administration in the rat using sucrose substitution in a sipper-tube procedure. Psychopharmacology, 147, 274279.

Schenk, S., Lacelle, G., Gorman, K., \& Amit, Z. (1987). Cocaine self-administration in rats influenced by environmental conditions: Implications for the etiology of drug abuse. Neuroscience Letters, 81, 227-231.

Schneider, M.L. (1992). Prenatal stress exposure alters postnatal behavioral expression under conditions of novelty challenge in rhesus monkey infants. Developmental Psychobiology, 25, 529-540.

Schneider, M.L., \& Coe, C.L. (1993). Repeated social stress during pregnancy impairs neuromotor development of the primate infant. Developmental and Behavioral Pediatrics, 14, 81-87).

Schneider, M.L., Moore, C.F., Kraemer, G.W., Roberts, A.D., \& DeJesus, O.T. (2002). The impact of prenatal stress, fetal alcohol exposure, or both on development: Perspectives from a primate model. Psychoneuroendocrinology, 27, 285-298. 
Setlow, B., Mendez, I.A., Mitchell, M.R., \& Simon, N.W. (2009). Effects of chronic administration of drugs of abuse on impulsive choice (delay discounting) in animal models. Behavioural Pharmacology, 20, 380-389.

Sinha, R. (2001). How does stress increase risk of drug abuse and relapse? Psychopharmacology, $158,343-359$.

Talge, N., Neal, C., \& Glover, V. (2007). Antenatal maternal stress and long-term effects on child neurodevelopment: How and why? Journal of Child Psychology and Psychiatry, 48, (245-261).

Taylor, J., Harris, N., \& Vogel, W.H. (1990). Voluntary alcohol and cocaine consumption in "low" and "high" stress catecholamine responding rats. Pharmacology, Biochemistry, and Behavior, 37, 359-363.

Thanos, P.K, Volkow, N.D., Freimuth, P., Umegaki, H., Ikari, H., Roth, G., et al. (2001). Overexpression of dopamine D2 receptors reduces alcohol self-administration. Journal of Neurochemistry, 78, 1094-1103.

Thomas, M.B., Hu, M., Lee, T.M., Bhatnagar, S., \& Becker, J.B. (2009). Sex-specific susceptibility to cocaine in rats with a history of prenatal stress. Physiology and Behavior, 97, 270-277.

Tolliver, G.A., Sadeghi, K.G., \& Samson, H.H. (1988). Ethanol preference following the sucrose-fading initiation procedure. Alcohol, 5, 9-13.

Vallee, M., Maccari, S., Dellu, F., Simon, H., Le Moal, M., \& Mayo, W. (1999). Long-term effects of prenatal stress and postnatal handling on age-related glucocorticoid secretion and cognitive performance: a longitudinal study in the rat. European Journal of Neuroscience, 11, 2906-2916. 
Vallee, M., Mayo, W., Dellu, F., Le Moal, M., Simon, H., \& Maccari, S. (1997). Prenatal stress induces high anxiety and postnatal handling induces low anxiety in adult offspring: Correlation with stress-induced corticosterone secretion. The Journal of Neuroscience, 17, 2626-2636.

Van den Bergh, B.R., Mennes, M., Oosterlaan, J., Stevens, V., Stiers, P., Marcoen, A., et al. (2005). High antenatal maternal anxiety is related to impulsivity during performance on cognitive tasks in 14- and 15-year-olds. Neuroscience and Biobehavioral Reviews, 29, 259-269.

Ward, H.E., Johnson, E.A., Salm, A.K., \& Birkle, D.L. (2000). Effects of prenatal stress on defensive withdrawal behavior and corticotrophin releasing factor systems in rat brain. Physiology \& Behavior, 70(3-4), 359-366.

Weinstock, M. (1996). Does prenatal stress impair coping and regulation of hypothalamicpituitary-adrenal axis? Neuroscience and Biobehavioral Reviews, 21, 1-10.

Weinstock, M., Matlina, E., Maor, G.I., Rosen, H., \& McEwen, B.S., (1992). Prenatal stress selectively alters the reactivity of the hypothalamic-pituitary-adrenal system in the female rat. Brain Research, 595, 195-200.

Welberg, L.A.M., \& Seckl, J.R. (2001). Prenatal stress, glucocorticoids and the programming of the brain. Neuroendocrinology, 13, 113-128.

West, C.H. \& Weiss, J.M. (2006). Intake of ethanol and reinforcing fluids in rats bred for susceptibility to stress. Alcohol, 38, 13-27.

White, M.J., Lawford, B.R., Morris, C.P., \& Young, R.M. (2009). Interaction between DRD2 C957T polymorphism and an acute psychosocial stressor on reward-related behavioral impulsivity. Behavioral Genetics, 39, 285-295. 